\title{
Checklist de Caelifera (Acridoidea e Eumastacoidea) do Estado do Rio de Janeiro (Orthoptera)
}

\author{
Cristiane Vieira Assis-Pujol1 ${ }^{\bowtie}$ \& José Roberto Pujol-Luz²
}

1. Universidade Católica de Brasilia, e-mail: cristianev@ucb.br (Autor para correspondência ${ }^{\bowtie}$ ). 2. Universidade de Brasília, Instituto de Biologia, e-mail: jrpujol@unb.br.

\section{EntomoBrasilis 7 (2): 134-15o (2014)}

Resumo. Este trabalho apresenta um checklist da fauna de Orthoptera, Caelifera (Acridoidea e Eumastacoidea), do estado do Rio de Janeiro, Brasil. Este artigo foi baseado no material depositado na Coleção de Orthoptera, Caelifera do Museu Nacional do Rio de Janeiro e em citações da literatura pertinente. Os resultados da pesquisa demonstram que os Caelifera do estado do Rio de Janeiro estão representados por duas superfamílias, Acridoidea e Eumastacoidea, quatro famílias (Proscopiidae, Ommexechidae, Acrididae e Romaleidae), subdivididos em 10 subfamílias, 16 tribos, 46 gêneros, 48 espécies e oito subspécies. Neste trabalho estão incluídas as listas de sinonímias para cada espécie, distribuição geográfica, localização dos tipos e principais referências bibliográficas.

Palavras-chave: Brasil; Distribuição Geográfica; Holótipos; Lista de Espécies; Taxonomia.

Checklist of Caelifera (Acridoidea and Eumastacoidea) of Rio de Janeiro State (Orthoptera)

Abstract. This work presents a checklist of the fauna of Orthoptera, Caelifera (Acridoidea and Eumastacoidea), the state of Rio de Janeiro, Brazil. This article is based on material deposited in the Collection of Orthoptera, Caelifera the National Museum of Rio de Janeiro, and citations of relevant literature. The survey results show that Caelifera the state of Rio de Janeiro are represented by two superfamilies, and Acridoidea and Eumastacoidea, four families (Proscopiidae, Ommexechidae, and Romaleidae Acrididae), subdivided into 10 subfamilies, 16 tribes, 46 genera, 48 species and eight subspecies. This work includes the lists of synonyms for each species, geographical distribution, types and location of primary references.

Keywords: Brazil; Geographical Distribution; Holotypes; List of Species; Taxonomy.

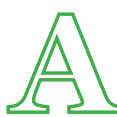
superfamília Acridoidea (Orthoptera, Caelifera), está distribuídaemtodasasregiõesbiogeográficas(Acridoidea), com exceção da Antártida e do Cículo Polar Ártico. Já os representantes da superfamília Eumastacoidea, possuem registro de ocorrência em toda a região tropical e subtropical, parte da Ásia e Oceania. Entretanto, a família Proscopiidae ocorre somente na America do Sul e sul da America Central (EADEs et al. 2012). Cerca de 10.000 espécies de ambas as superfamílias estão descritas no mundo e aproximadamente 1.000 espécies são conhecidas no Brasil, amplamente distribuídas por todo o território brasileiro (GALLO et al. 2002). Representantes destas superfamílias destacam-se, principalmente, por sua importância econômica, devido aos enormes prejuízos que algumas de suas espécies causam aos cultivos, podendo vir a devastar lavouras inteiras (GALLO et al. 2002).

Com base na classificação de Flook \& Rowell, 1997, Foi elaborado um checklist das espécies que ocorrem no Estado do Rio de Janeiro. Complementam o trabalho a atualização taxonômica e geográfica das espécies, localização dos tipos e principais registros bibliográficos.

Dados gerais sobre a fauna de Caelifera (Acridoidea e Eumastoicoidea) do Rio de Janeiro. A fauna Acridoidea e Eumastacoidea do Estado do Rio de Janeiro é composta por 48 espécies e oito subespécies conhecidas, agrupadas em 41 gêneros, 11 subfamílias e quatro famílias (Proscopiidae, Ommexechidae, Romaleidae e Acrididae).

Para algumas espécies, só existem registros de ocorrência para o estado do Rio de Janeiro [Staleochlora trilineata (Serville,
1831), Tijucella polychroma Amedegnato \& Descamps, 1979, Deinacris picta picta Amedegnato \& Descamps, 1979, Seabracris tijuca Amedegnato \& Descamps, 1979, Beckeracris janeirensis Amedegnato \& Descamps, 1979 e Orphula pagana (Stal, 1860)].

Uma nova ocorrência para Rhammatocerus pseudocyanipes Assis-Pujol, 1997 é incluída no trabalho.

\section{MATERIAIS E MÉTODOS}

A maior parte do material listado está depositada na coleção de Caelifera do Museu Nacional do Rio de Janeiro, que é proveniente de diversas coletas realizadas ao longo de anos pela equipe da instituição, bem como por doações. O restante do material que possui registro de ocorrência no Rio de Janeiro, porém, que não existem exemplares no Museu Nacional, foi incluído com base em registros na literatura disponível.

Classificação adotada é a proposta por FLOOK \& Rowell (1997) e por EADEs \& OTTE (2014), para as tribos e grupos. As espécies que apresentam * são as espécies-tipo dos respectivos gêneros. Os nomes em negrito correspondem aos nomes válidos e os subsequentes, em itálico, são sinônimos.

Locação dos tipos. A locação dos tipos das espécies estudadas está indicada no texto. A Tabela 1 apresenta o número de tipos depositados em cada instituição. Os nomes correspondentes às siglas e a localização das instituições são os seguintes: 
Tabela 1. Número de tipos depositados em cada instituição de pesquisa. O número 1 seguido e ponto de interrogação no NHMW e MIZT, corresponde à dúvida na literatura com relação à locação do tipo de Bucephalacris bucephalus.

\begin{tabular}{ccccccccccccccc}
\hline \multicolumn{11}{c}{ Instituição depositária } \\
\hline & ANSP & BMNH & MHNG & MIZT & MNHN & MNRJ & NHMW & NHRS & RMNH & USNM & UZIU & ZMHB & ZSMC & Perdidos \\
\hline $\begin{array}{c}\text { Tipos } \\
\left(\mathrm{n}^{\circ}\right)\end{array}$ & 3 & 4 & 2 & $3+(1$ ?) & 5 & 9 & $4+(1 ?)$ & 7 & 1 & 2 & 7 & 2 & 2 & 3 \\
\hline
\end{tabular}

- $\quad$ ANSP - Academy of Natural Sciences of Philadelphia, Pennsylvania, United States.

- $\quad$ BMNH - The Natural History Museum, London, Unites Kingdom.

- MHNG - Muséum d'Histoire Naturelle, Geneva, Switzerland.

- $\quad$ MIZT - Universitá di Torino, Torino, Italia.

- MNHN - Muséum National d`Histoire Naturelle, Paris, France.

- $\quad$ MNRJ - Museu Nacional, Universidade Federal do Rio de Janeiro, Rio de Janeiro, Rio de Janeiro, Brasil.

- NHMW - Naturhistorisches Museum, Wien, Áustria.

- $\quad$ NHRS - Naturhistoriska Riksmuseet, Stockholm, Sweden.

- RMNH - Nationaal Natuurhistorisch Museum, Leiden, Netherlands.

- USNM - National Museum of Natural History, Washington, D.C., United States.

- $\quad$ UZIU - Uppsala University, Uppsala, Sweden.

- ZMHB-Museum für Naturkundeder Humboldt-Universität, Berlin, Germany.

- ZSMC-ZoologischeStaatssammlungdês Bayerischenstaates, Munichen, Germany.

\section{RESULTADOS}

\section{LISTA DAS ESPÉCIES ENCONTRADAS NO RIO DE JANEIRO}

\section{ACRIDOIDEA}

\section{OMMEXECHIDAE}

\section{Ommexechinae}

\section{Ommexecha Serville 1831: 285}

* virens Serville 1831: 286

germari Burmeister, 1838: 655

servillei Blanchard, 1836: 613

Distribuição geográfica: Argentina (Buenos Aires, Corrientes, Misiones, Chaco, Entre Rios, Santa Fé, Formosa); Uruguai; Paraguai (Paraguari, San Pedro, Alto Paraná, Itapua, Guairá); Bolívia (Santa Cruz, Cochabamba); Brasil (RS, PR, SC, SP, RJ, ES, BA, PE, CE, MG, MT, GO).

Tipo: MNHN [Holótipo macho. Prov. Corrientes (Ommexecha servillei)].

Refs.: Serville (1831: 286, 1839: 696); Blanchard (1836: 312); Bolívar (1884: 29); BRUNER (1900: 47, 1906: 639); KIRBY (1910: 592); REHN (1907: 168, 1913: 273); LiEBERMANN (1937: 55, 1939: 165, 1941: 41; 1948: 55, 1958: 3, 1967: 503); SilveIRA-Guido et al. (1958: 485); RoNDEROS (1977: 101, 1978: 97); C.O.P.R. (1982:
96); CARVAlHo et al. (2011: 123).

\section{ROMALEIDAE}

\section{Romaleinae}

Romaleini

\section{Agriacris Walker 1870: 644}

Camposia Bolívar, 1909: 346

Elaeochlora Stål, 1873: 52

auripennis (Walker) 1870: 522. (Xiphocera)

hilaris Gerstaecker, 1889: 10

hymenaea Gerstaecker, 1873: 186

parvispina Pictet \& Saussure, 1887: 344

psittacina Gerstaecker, 1973: 187

scabra Thunberg, 1824: 400

spoliata Walker, 1870: 524

Distribuição geográfica: Brasil (AM, PA, PB, PE, RO, MG, ES, RJ, SP); Peru (Huanuco, Cusco, Loreto).

Tipo: BMNH [Holótipo fêmea. “Amer. Merid.”].

Refs.: Walker (1870: 522); Pictet \& SAUSSURe (1887: 346); Kirby (1910: 366); LiEBERMANN (1969: 85); RoberTs \& CARBONELL (1992: 82).

\section{Chromacris Walker 1870: 643}

* speciosa (Thunberg) 1824: 404 (Gryllus)

stolli Pictet \& Saussure, 1887: 351

xanthoperum de Haan 1835: t.a, fig. 2

Distribuição geográfica: Brasil (AP, PA, AM, BA, PE, ES, RJ, MG, SP, PR, SC, RS, MT, MS); Colômbia (Magdalena); Venezuela (Carabobo, Cojedes); Guiana; Equador (Bolívar); Peru (Junin, Pasco, Cusco); Bolívia (Santa Cruz); Paraguai (Guaira, Paraguari); Argentina (Salta, Misiones, Catamarca, Cordoba, Entre Rios, Buenos Aires); Uruguai (trogon trogon) (GERSTAECKER 1873) (Romalea); Costa Rica; (?)Nicaragua.

Tipo: UZIU [Lectótipo fêmea. Brasil (sem localidade exata)].

Refs.: Thunberg (1824: 404); WALKer (1870: 644); Hudson (1895: 127); LiEBERMANN (1935: 119); HEPPER (1945: 280); BARRERA \& Paganini (1975: 107); Turk et al. (1976: 6); VirLA DE ARguello (1978: 113); RoberTs \& CARBONELl (1982: 51); C.O.P.R. (1982: 104); Riede (1987: 277); CARBONELl et al. (2006); CARBOnELl et al. (2007: 45); BuzetTI \& CARotTi (2008: 48); ARAújo Cisneiros et al. (2012: 1); BidAu (2012: 256); BARRIENTOS LozANo et al. (2013: 319).

\section{Procolpia Stål 1873: 52}

* emarginata (Serville) 1831: 271 (Xiphicera). 
Distribuição geográfica: Brasil (?) (BA, SP, RJ); Bolívia (Santa Cruz); (?) Venezuela; (?) América Central; (?) México.

Tipo: MNHN [Holótipo fêmea. Brasil (sem localidade exata)].

Refs.: Serville (1831: 271); Scudder (1869: 84); Stal (1873: 52); Pictet \& SAussure (1887: 340); Bolívar (1896: 16); Bruner (1905-1909: 223, 1919: 36); KirBy (1910: 364); HEBARD (1932: 268); REHN (1955: 41).

\section{Staleochlora Roberts \& Carbonell 1992: 91.}

* trilineata (Serville) 1831: 272 (Xiphicera)

Distribuição geográfica: Brasil (RJ).

Tipo: MNRJ [Neótipo macho designado por RoBERTS \& CARbonell (1992), Floresta de Tijuca, Rio de Janeiro, Brasil].

Refs.: Serville (1831: 272); Burmeister (1861: 491); Walker (1870: 521); Stal (1873: 46); Pictet \& SAussure (1887: 344); Giglio-Tos (1897: 29); ReHN (1909: 124); KirBy (1910: 365); BRUNER (1911: 54); LiEBERMANN (1935: 41, 1939: 177, 1948: 80, 1969: 85); C.O.P.R. (1982: 108); MESA et al. (1982: 516); RIEDE (1987: 277); Roberts \& CARBonell (1992: 100); Donato (2000: 80); Mesa et al. (2004: 15).

\section{Titanacris Scudder 1869: 352}

Lophacris Scudder 1869: 352

olfersii (Burmeister) 1838: 628. (Acridium)

flavicornis Stoll 1813: 19, pl. 8b.

semirubrum Serville 1839: 653

Distribuição geográfica: Brasil (RJ, BA, ES).

Tipo: MNRJ [Neótipo macho designado por DESCAMPS \& Carbonell (1985), Fl. da Tijuca, Rio de Janeiro, Brasil].

Refs.: Burmeister (1838: 628); Pictet \& SAussure (1887: 363); LIEBERMANN (1939b: 598); HAYWARD (1860: 78); DESCAMPS \& Amedegnato (1970: 863); Walker (1870: 549); Hebard (1923: 232); Dirsh (1956: 275); Descamps \& Carbonell (1985: 270).

\section{Tropidacris Scudder 1869: 338}

Eutropidacris Hebard 1923: 232

cristata grandis (Thunberg) 1824: 403

fabricii Scudder, 1869: 394

Distribuição geográfica: Brasil (BA, ES, RJ, SP, PR, SC, RS); L. Paraguai; Argentina (Misiones).

Tipo: UZIU [Holótipo fêmea. "South America, America Meridionalis"].

Refs.: Thunberg (1824: 403); STÅL (1873: 49); Pictet \& SAussure (1887: 361); REHN (1909: 134); KIRBY (1910: 380); LIEBERMANN (1939: 597; 1955: 336); C.O.P.R. (1982: 118); CARBONELl (1986: 366); CARBOnell et al. (2006: CD Rom).

collaris (Stoll) 1813: 39 pl.31, fig.80 (Gryllus locusta)

carinatus Thunberg, 1815: 222

rugosus Thunberg, 1824: 401

Distribuiçãogeográfica: Colômbia (Bogotá, Guajira, Magdalena, Cordoba, Santander, Cundinamarca); Venezuela (Falcon, Zulia, Nueva Esparta, Distrito Federal, Miranda, Guarico, Monagas, Bolívar); Guiana; Suriname; Guiana Francesa; Peru (Loreto, San Martin); Bolívia (Beni, Santa Cruz); Brasil (RR, AM, PA,
MA, RO, MT, MS, RN, PB, PE, BA, MG, GO, ES, RJ, SP, PR, SC, RS); Argentina (Catamarca, Jujuy, Salta, Tucuman, La Rioja, Cordoba, San Luis).

Tipo: RMNH [Holótipo fêmea, Bogotá (provavelmente)].

Refs.: Stoll (1813: 39); Kirby (1910: 379); RehN (1916: 284); Hebard (1923: 233); Liebermann (1939b: 594); PizA JR. (1958: 247); Hayward (1960: 3); Descamps \& Amedegnto (1970: 870);

BarRera \& PAGANini (1975: 107); GangWere \& Ronderos (1975: 173); Barrera \& Turk (1977: 167); Descamps (1979: 313); C.O.P.R. (1982: 110); MARShall (1983: 386); CARBONELl (1984: 8, 1986: 390); CARbonell et al. (2006: CD Rom); CARbonell et al. (2007: 46).

\section{Zoniopoda Stål 1873: 32, 51}

* tarsata (Serville), 1831: 283 (Acridium)

cruentata Blanchard, 1837: 216

Distribuição geográfica: Brasil (ES, RJ, SP, PR, SC, RS); Argentina (Centro e norte do país); Paraguai (Central, Paraguari); Uruguai.

Tipo: MNHN [Holótipo fêmea. Brasil (sem localidade exata)].

Refs.: Serville (1831: 283); Pictet \& SAussure (1887: 356); Hudson (1895: 29); Giglio-Tos (1897: 29); ReHN (1908: 16); KIRBY (1910: 375); BRUNER (1911: 59, 1919: 38); LIEBERMANN (1928: 137, 1939: 182, 1948: 55, 1949a: 1, 1949b: 14, 1950: 16, 1951: 39, 1954, 1958: 3, 1972a: 191, 1972b: 6); HeBARD (1931: 257); Blanchard (1945: 280); Hepper (1945: 23); LiebermanN \& Schiuma (1946: 1); Schiuma (1951: 10); Hayward (1960: 3); Greathead (1963: 437); CAMPODONico \& SANTORO (1971: 63); Santoro \& Carames (1973: 197); Gangwere \& Ronderos (1975: 173); BARRERA \& PAGANINI (1975: 107); SANTORO (1976: 1); BARRERA \& TURK (1977: 167); C.O.P.R. (1982: 125); Cigliano et al. (2000: 81); CARBONELl et al. (2006: CD Rom); CARBONELl et al. (2007: 4); PARAdEda et al. (2008: 123); BidAu (2012: 256).

\section{ACRIDIDAE}

\section{Melanoplinae}

\section{Dichroplini}

\section{Baeacris Rowell \& Carbonell 1977: 56}

punctulatus (Thunberg), 1824: 408 (Dichroplus)

Distribuição geográfica: Brasil (RR, AM, PA, PE, MT, MG, ES, RJ, SP); Bolívia (Santa Cruz); Paraguai (Caaguazu); Argentina; Uruguai; L.Bolívia; L.Peru; Equador, Colômbia; Venezuela; Guiana.

Tipo: UZIU [Holótipo macho. Brasil (sem localidade exata)].

Refs.: Thunberg (1824: 408); BERG (1881: 38); Giglio-Tos (1897: 34, 1898: 67); REHN (1907: 188, 1913: 343); BRUNER (1910: 487, 1919: 84); HEBARD (1923: 285, 1924: 193); RoBERTS (1937: 357); LiebermanN (1939a: 211); CARbonell \& Ronderos (1973: 366); BarRera \& TURK (1977: 167); Virla de Arguello (1978: 113); ESCALANTE (1979: 113); VillaLobos (1980: 325); LunA et al. (1981: 243); C.O.P.R. (1982: 163); RiEdE (1987: 286); BeNTOS-PEREIRA (1989: 31); Cigliano (1991: 176); Ronderos \& Cigliano (1991: 176); Cigliano et al. (1995: 41); Michel \& Teisaire (1996: 29); Lange (1997: 63); Cigliano \& Lange (1998: 67); Cigliano et al. (2000: 81); DE WysiecKi et al. (2004: 87); CоLOMBo et al. (2005: 375); CARBonell et al. (2006: CD Rom); CARBonell et al. (2007: 49); BuzzetTI \& CARotTI (2008: 51); Dias-Guerra et al. (2010: 333); MARIOTTINI et al. (2011: 737). 


\section{Dichroplus Stal 1973: 78}

Trigonophymus Stal 1878: 32

fuscus (Thunberg) 1815: 235 (Gryllus)

Distribuição geográfica: Brasil (RJ, ES, BA, PE, MG, RS); Argentina (Santiago del Estero, Chaco); Paraguai (Caaguazu); Bolívia (Cochabamba).

Tipo: UZIU [Holótipo fêmea com etiqueta "fuscus - Nov. Cambr.”].

Refs.: Thunberg (1815: 235); StÅl (1873: 78, 1978: 6); GiglioTos (1894: 21, 1897: 34, 1898: 67); PRINZESSIN VON BAYERN (1900: 257); Kirby (1910: 487); LiebERMANN (1939a: 21, 1965: 216); Ronderos et al. (1968: 271); CARBONELl et al. (2006: CD Rom); BuZzETTI \& CAROTTI (2008: 52).

\section{Digamacris Carbonell 1989: 77}

* amoena (Stål) 1878: 8 (Pezotettix)

Distribuição geográfica: Brasil (RJ, SP).

Tipo: NHMW [Holótipo fêmea. "Brasil. Boucard ded.”]

RefS.: STÅL (1878: 8); SCUDdER (1897: 406); BRUNER (1900: 78); Kirby (1910: 490); REHN (1913: 113); LiEBERMANN (1929: 180, 1971: 146); CARBONELL (1989: 80).

fraternus (Carl) 1916: 514 (Dichroplus)

Distribuição geográfica: Brasil (MG, ES, RJ).

Tipo: MHNG [Minas Gerais, Brasil].

Refs.: CARL (1916: 514); LIEBERMANN (1955: 343, 1968: 29); REHN (1913: 113); CARBONELL (1989: 84); Hollier (2010: 29).

\section{Leiotettix Bruner 1906: 684.}

* viridis Bruner 1906: 685

punctipes Bruner, 1906: 686

Distribuição geográfica: Paraguai (Paraguari); Brasil (MT, MG, RJ, RS); Bolívia (Santa Cruz); Argentina (Misiones).

Tipo: USNM [Síntipo Sapucai, Paraguai].

Refs.: BRUNeR (1906: 685, 1919: 85); ReHN (1907: 188); KiRBY (1910: 490); LiEBERMANN (1939a: 214，1965: 219); Uvarov (1966: 179); Mesa \& Zolessi (1968: 4); C.O.P.R. (1982: 168); Cigliano \& Ronderos (1994: 148); Ronderos \& CARBonell (1994: 168); Cigliano et al. (1996: 125); Соцомвo et al. (2005: 375); CARBonell et al. (2006: CD Rom); CHAPCO (2006: 59).

\section{Ronderosia Cigliano 1997: 19}

bergi (Stål) 1878: 6 (Dichroplus)

brasiliensis Bruner, 1906: 682

Distribuição geográfica: Brasil (RJ, BA, ES, SP, PN, SC, RS); Paraguai (Paraguari, Alto Parana, Caaguazu); Uruguai; Argentina (Buenos Aires, Entre Rios, Corrientes, Santa Fé, Cordoba, Chaco, Salta, Missiones).

Tipo: NHRS [Holótipo macho. Buenos Aires, Argentina].

Refs.: StÅL (1878: 6); Giglio-Tos (1894: 21); Rehn (1905: 41, 1907: 188, 1913: 345); Kirby (1910: 489); LiEBERMANN (1939: 208); Ronderos (1964: 82); DiRsh (1965: 41); C.O.P.R. (1982: 151); Bentos-Pereira (1989: 31); Cigliano (1997: 3); Cigliano \& LANGe (1998: 67); Cigliano et al. (2000: 81); DE Wysiecki et al. (2004: 87); CоLомво et al. (2005: 375); CARBONELl et al. (2006:
CD Rom); CHAPCO (2006: 59); MARIOTTINI et al. (2006: 81, 2010: 92); CASTILlo et al. (2010: 775).

\section{Parascopini}

\section{Tïjucella Amedegnato \& Descamps 1979: 485}

* polychroma Amedegnato \& Descamps 1979: 486

Distribuição geográfica: Brasil (RJ).

Tipo: MNRJ [Holótipo macho. Fl. da Tijuca, Rio de Janeiro, Brasil].

Refs.: Amedegnato \& Descamps (1979: 486); Ronderos (1985: 54); Отте (1995: 413); Yin et al. (1996: 703).

\section{Proctolabinae}

Proctolabini

Proctolabae

\section{Poecilocloeus Bruner 1910: 174}

Diadelacris Descamps, 1976: 64, 70, 126

janeirensis Descamps 1980: 169.

Distribuição geográfica: Brasil (RJ, PR).

Tipo: MNRJ [Holótipo macho. Fl. da Tijuca, Rio de Janeiro, Brasil; Alótipo fêmea: mesma localidade].

Refs.: Descamps (1980: 169); Amedegnato \& Descamps (1982: 163).

\section{Copiocerinae}

\section{Aleuasini}

\section{Zygoclistron Rehn 1905: 39}

Nuceria Stål 1878: 23, preoc.

Nuciera Bolivar, 1906: 96

falconinum (Gerstaecker) 1873: 191 (Acridium)

acutum Rehn, 1913: 96

roseipennis Stål, 1978: 61

Distribuição geográfica: Brasil (RJ, MG).

Tipo: ZMHB [Holótipo macho. Rio de Janeiro, Brasil].

Refs.: Gerstaecker (1873: 191); Kirby (1910：419); Carbonell (1969: 580).

\section{Copiocerini}

\section{Copiocerae}

\section{Bucephalacris Giglio Tos 1894: 30}

Chrysopsacris Bruner 1908: 215, 282

Paraleuas Giglio Tos 1898: 47, 57

* bucephalus (Marschall) 1836: 217 (Gryllus)

minor Bruner, 1906: 669

punctipennis Bruner, 1906: 669

Distribuição geográfica: Brasil (BA, AM, MG, RJ, MT); Paraguai (Paraguari, Caaguazu). 
Tipo: NHMW ou MIZT [Holótipo macho Bahia, Brasil].

Refs.: Marschall (1836: 217); Giglio-Tos (1894: 30); Bruner (1908: 282); Descamps (1977: 64); Passerin d’Entreves (1981: 35).

Copiocera Burmeister 1838: 611 (= Glaphyracris Walker, 1870: 653)

* erythrogastra (Perty) 1834: 122 (Xiphicera)

eucera (Marschall) 1835: 216

Distribuição geográfica: Brasil (MG, RJ, MT).

Tipo: ZSMC [Lectótipo fêmea. "in montibus Prov. Minarum”].

Refs.: Perty (1834: 122); Burmeister (1838: 612); Bruner (1911: 89); REHN (1916: 289, 1920: 249); HEBARD (1924: 121); Descamps (1983: 293); Descamps (1984: 36); Buzetti \& CarotTi (2008: 53).

\section{Episcopotettix Rehn 1902: 13}

Rehnacridium Costa-Lima 1942: 64

* sulcirostris Rehn 1902: 13

incognitum Costa-Lima, 1942: 64

Distribuição geográfica: Brasil (RJ, GO, MT, BA); Argentina (Misiones).

Tipo: ANSP [Holótipo macho. Forest of San Juan, Mexico].

Refs.: ReHN (1902: 13, 1920: 249, 1955: 34); LIEBERMANN (1947: 393, 1955: 339); Costa-Lima (1942: 64); Descamps (1984: 14); CARbonell et al. (2006: CD Rom).

\section{Opshomala Serville 1831: 267 (Opomala auct.)}

Chrostheippus Bruner, 1911: 98

* viridis Serville 1831: 267

varipes Serville, 1839: 588

Distribuição geográfica: Brasil (RJ, BA, ES).

Tipo: perdido. Localidade desconhecida para O.viridis e errada (America Septentrional) para O.varipes.

Refs.: SERVILLE (1831: 267, 1839: 588); BurMEISTER (1838: 610); BRUNER (1908: 255, 1911: 89); KIRBy (1910: 419); REHN \& EADES (1961: 7); Descamps (1984: 17).

\section{Monachidiae}

\section{Antiphon Stål 1878: 55 .}

(*) acropyrinon (Perty) 1834: 132. (Monachidium)

* gallus Stål 1878: 55

Distribuição geográfica: Brasil (RJ, SP, PN, SC).

Tipo: ZSMC [Lectótipo fêmea. "in Provincia Bahiense” Brasil].

Refs.: PERTY (1834: 132); STÅL (1878: 55); Kirby (1910: 464); LiebermanN (1940: 363); DesCAMPs (1983: 292, 1984: 50).

\section{Leptysminae}

\section{Leptysmini}

Belosacris Rehn \& Eades 1961: 111

(*) coccineipes (Bruner) 1906: 659 (Arnilia)
* aurantipes Rehn \& Eades, 1961: 113

intermedia Bruner, 1919: 46

Distribuição geográfica: México (Veracruz); Panamá; Venezuela (Guarico); Trinidad; Guiana; Suriname; Guiana francesa; Brasil (AP, PA, BA, MG, RJ, SP, MT, MS); Paraguai (Paraguari, Central); Bolívia (Santa Cruz, Beni); Argentina (Misiones, Chaco); Uruguai.

Tipo: USNM [Sapucai, Paraguai].

Refs.: BRUNER (1906: 659, 1919: 46); REHN (1907: 180); REHN \& EADES (1961: 111); RoBERTS (1977 [1978]: 56); C.O.P.R. (1982: 182); Bentos-Pereira \& Lorier (1991: 631); Carbonell et al. (2006: CD Rom);

\section{Cylindrotettix Bruner 1906: 156}

obscurus (Thunberg), 1827: 77 (Truxalis)

Distribuição geográfica: Brasil (PA, MT, CE, PE, BA, MG, RJ, SP, SC); Colômbia (Meta, Vaupes, Putumayo); Peru.

Tipo: UZIU [Guanabara (Rio de Janeiro), Brasil].

Refs.: Thunberg (1827: 77); SCUdDER (1869: 83); BRUNer (1895: 64, 1906: 12, 1911: 72); ReHN (1905: 804, 1907: 180, 1913: 336, 1915: 270); HEBARD (1931: 273); LiEBERMANN (1939: 125); REHN \& EAdes (1961: 81); RoberTs (1975: 37); Descamps (1976: 291); BeINGOlEA GUERRERO (1989: 38); OTTE (1995: 55); CARBONELl et al. (2006: CD Rom); CARBONELl et al. (2007: 52).

\section{santarosae Roberts 1975: 36}

Distribuição geográfica: Brasil (MT, PA, GO, MG, ES, RJ, SP, SC); Paraguai (Guairá); Bolívia (Santa Cruz); Argentina (Chaco, Misiones); Uruguai.

Tipo: ANSP [Holótipo macho. Sta Rosa dos Descalvados, Mato Grosso, Brasil].

Refs.: Roberts (1975: 36); Donato (2000: 78); CARbonell et al. (2006: CD Rom)

\section{Leptysma Stål 1873: 42, 85 .}

filiformis (Serville) 1839: 591 (Opsomala)

gracilis Bruner, 1906: 58

minima Bruner, 1906: 58

Distribuição geográfica: Brasil (SP, RR, PA, BA, MG, ES, RJ, PR, GO, MT); Venezuela (Carabobo, Bolivar, Barinas); Colômbia (Meta); Trinidad; Guiana; Suriname; Paraguai (Paraguari); Bolívia (Santa Cruz); Uruguai.

Tipo: MNHN [Holótipo fêmea. São Paulo, Brasil].

Refs.: Serville (1839: 591); Berg (1881: 37); Bolívar (1888: 146); Giglio-Tos (1897: 30); BrunER (1906a: 613, 1906b: 135, 1911: 72, 1919: 43); ReHN (1907: 180, 1916: 285); REHN \& EADES (1961: 85); RoBERTS (1977: 44); DesCAMPs (1979: 314); C.O.P.R. (1982: 186); CARBonell et al. (2006: CD Rom); CARBonell et al. (2007: 52).

\section{Stenacris Walker 1870: 65}

Arnilia Stål, 1873: 42, 85

xanthochlora (Marschall) 1836: 215 (Gryllus)

cayennensis Bruner, 1919: 50

concolor Walker, 1870: 652 
cylindrodes Stål, 1860: 325

interior Bruner, 1908: 254

lanceolata Walker, 1870: 651

reyesi Descamps, 1975: 65

saussurei Carl, 1916: 510

Distribuição geográfica: Brasil (RJ, AP, PA, Ceará, PE, BA, MG, ES, SP, GO, SC, RS, MT); México (Veracruz); Guatemala; Costa Rica; Panamá; Colômbia (Magdalena, Meta); Venezuela; Trinidad; Suriname; Guiana Francesa; Equador (NapoPastaza); Paraguai (Guayra); Bolívia (Beni, Santa Cruz, Tarija); Argentina (Formosa, Chaco, Misiones).

Tipo: NHMW [Holótipo macho. "Near Rio de Janeiro, Brasil”].

Refs.: Marschall (1836: 215); WALKER (1870: 651); Giglio-ToS (1894: 1); BRUNER (1900: 80, 1919: 50); REHN (1915: 270, 1916: 285, 1918: 170); CARL (1916: 510); LiEBERMANN (1928: 137, 1939: 125); RoBERTS (1937: 356, 1977[1978]: 61); REHN \& EADES (1961: 81); Descamps (1975: 61); Bentos-Pereira \& Lorier (1991: 631); Carbonell et al. (2006: CD Rom); Carbonell et al. (2007: 52); BuzETTI \& CAROTTI (2008: 54).

\section{Tetrataeniini}

\section{Cornops Scudder 1875: 276}

Paracornops Giglio Tos, 1894: 31

aquaticum (Bruner) 1906: 663

pelagicum Bruner, 1922: 61

politum (Bruner, 1906): 664

scudderi Bruner, 1906: 12

Distribuição geográfica: Brasil (AM, AP, PA, MT, GO, MG, RJ, SP, MS ); México; Panamá; Guatemala; Belize, Honduras; Trinidad \& Tobago; Nicarágua; Colômbia; Venezuela; Paraguai; Suriname; Guyana; Peru; Uruguai; Argentina; Bolívia; Equador.

Tipo: NHMW [Holótipo macho. [Paraguai, San Bernardino].

Refs.: BRUNER (1906: 663, 1919: 61); LiEBERMANN (1939: 125, 1941: 42, 1943: 22, 1948: 55, 1951: 39, 1958: 3); HePPER (1945: 280); Zolessi (1956: 3); BenNetT \& ZwolfER (1968: 832); Bennett (1970: 10); Mitchell \& Thomas (1972: 50); SilveiraGuido (1975: 400); Guido \& Perkins (1975: 400); ANdres \& BENNET (1975: 20); GANGWERE \& Ronderos (1975: 173); RobERTS \& CARBONELL (1979: 127); CARBONELl (1981: 92); C.O.P.R. (1982: 183); Braker (1989: 389); Adis \& Junk (1990: 349); BentosPereira \& Lorier (1991: 631); Cilliers (1991: 207); Turk (1993: 463); SPERBER \& LOPES (1995: 21); TURK \& Aquino (1996: 427); DOS Santos \& Vieira (1999: 37); Hill \& Cilliers (1999: 103); Hill \& ObERHOLZER (2000: 349); FERREIRA \& VASCONCELLOS-NeTO (2001: 523); Oberholzer \& Hill (2001: 82); Marques et al. (2002: 6); Adis \& JUNK (2003: 245); VieIra \& DOS SANTOS (2003: 711); Adis et al. (2004: 127); LHANO et al. (2005: 397); FRANCESCHINI et al. (2005: 373); CARBONELl et al. (2006. CD Rom); CARBONELl et al. (2007: 52); Adis et al. (2007: 12); CAPELLO et al. (2007: 209); FrANCESCHINI et al. (2007: 149); FonTANA et al. (2008: 154); DA Silva et al. (2010: 535); CAPELlo et al. (2011: 170); BRAGA et al. (2011: 185); FRANCESCHINI et al. (2011: 203); CAPELLO et al. (2012: 51)

$\left({ }^{*}\right)$ frenatum frenatum (Marschall) 1836:212 (Gryllus)

* bivittatum Scudder, 1875: 276 insularis Bruner, 1908: 262

lineatus Thunberg, 1824: 413

longicorne Bruner, 1911: 82

Distribuição geográfica: Brasil (RJ, PA, BA, ES, PR, MT); Colômbia (Meta); Venezuela (Aragua); Trinidad; Suriname; Guiana Francesa; Equador (Napo); Peru (Huanuco, Junin, Loreto, Cusco, Madre de Dios); Bolívia (Santa Cruz, Beni); Paraguai (Amambay).

Tipo: NHMW [Holótipo fêmea. "Provavelmente Rio de Janeiro, Brasil"].

Refs.: Marschall (1836: 212); Giglio-Tos (1898: 56); Bruner (1910: 472); Roberts \& CARBONELl (1979: 125); BRAKER (1989: 389); CARBONELl et al. (2007: 53); BRAGA et al. (2007: 227); AdIS et al. (2007: 18); BuzetTi \& CAROtTI (2008: 54); DE PAUlo Lemos et al. (2010: 43); RiBEIRo et al. (2013: 225).

\section{Stenopola Stål 1873: 83}

Heniola Uvarov, 1940: 174

Inusia Giglio Tos, 1897: 30

Oxyblepta Stål, 1873: 84

Pseudoxyblepta Descamps \& Amedegnato, 1972: 525

*dorsalis (Thunberg) 1827: 80 (Truxalis)

antillarum Rehn, 1908: 395

bicolor Bruner, 1908: 259

chipmani Bruner, 1906: 154

femoralis Walker, 1870: 509

flavipes Burmeister, 1838: 609

gracillima Giglio Tos, 1897: 31

inornatipes Bruner, 1908

janeirensis Bruner, 1908: 259

nana Bruner, 1908: 259

Distribuição geográfica: México (Tamaulipas, San Luis Potosi, Guerrero Tabasco Yucatan); Guatemala; Belize; Costa Rica; Panamá; Colômbia (Magdalena, Meta); Venezuela; Trinidad; Guiana; Suriname; Guiana Francesa; Brasil (PA, BA, MG, ES; RJ); Paraguai; L.Bolívia.

Tipo: UZIU [Holótipo macho. Brasil (sem localidade exata)].

Refs.: Thunberg (1827: 80); SCUdDER (1869: 83); Kirby (1890: 536); Giglio-Tos (1897: 31); Bruner (1919: 56); Roberts \& CARbonell (1979: 111); C.O.P.R. (1982: 189); CARBonell et al. (2006: CD Rom); CARBONELl et al. (2007: 53).

\section{puncticeps puncticeps (Stål) 1860: 325 (Opsomala)}

minor Bruner, 1919: 57

Distribuição geográfica: Brasil (BA, ES, RJ, PR, SC, RO, AC, MT, MS); Peru (Junin, Cusco); Bolíivia (Santa Cruz, Beni); Paraguai (Paraguari); Argentina (Formosa, Chaco, Misiones, Tucuman).

Tipo: NHRS [Holótipo macho. Brasil; R.F. Sahlberg col. 18491951 em viagem por Rio de Janeiro, Minas Gerais e Espirito Santo]. 
Refs.: STÅL (1861: 325); Giglio-Tos (1897: 31); Bruner (1900: 80, 1919: 57); REHN (1905: 38, 1913: 337); LIEBERMANN (1928: 137, 1939: 125, 1945: 235); RoBERTS \& CARBONELl (1979: 117); CARBonell et al. (2006: CD Rom).

\section{Ommatolampidinae \\ Pycnosarcini}

Pycnosarcus Bolivar 1906: 392 (Polysarcus Saussure 1859)

Alectrolophus Kirby 1914

Polychitonacris Rehn 1909

* atavus (Saussure) 1859: 393 (Polysarcus)

bimaculatus Kirby

Distribuição geográfica: Brasil (BA, MG, RJ)

Tipo: MHNG [Bahia, Brasil].

Refs.: SAussure (1859: 393); Rehn (1909: 163); Kirby (1910: 544); Kevan (1968: 66); OtTe (1995: 87); Hollier (2012: 305).

\section{Ommatolampidini}

\section{Ommatolampae}

\section{Vilernae}

\section{Vilerna Stål 1873: 38, 71}

rugulosa Stål 1878: 61

coriacea (Burmeister) 1838: 608, nomen oblitum

Distribuição geográfica: Peru (Junin, Loreto, Hunuco, Cusco, Madre de Dios); Colômbia (Vaupes, Putumayo, Amazonas); Venezuela (Bolívar); Equador; Brasil (AC, AM, BA, ES, GO, MT, MG, PA, PR, RO, RJ, SP); L. Bolívia; Paraguai; Argentina (Chaco, Salta).

Tipo: NHRS [Peru (sem localidade exata)].

Refs.: STÅL (1878: 61); Giglio-Tos (1894: 1); BolíVAR (1896: 16); BRUNER (1900: 80, 1911: 115); REHN (1907: 186); LIEBERMANN (1928: 137, 1939: 125, 1945: 3); HAYWARD (1960: 3); C.O.P.R (1982: 193); Descamps \& Amedegnato (1989: 25); Carbonell et al. (2007: 56); BuzETTI \& CAROTTI (2008: 57).

\section{Syntomacrini}

\section{Syntomacrae}

Deinacris Amedegnato \& Descamps 1979: 447.

*picta picta Amedegnato \& Descamps 1979: 449.

Distribuição geográfica: Brasil (RJ).

Tipo: MNHN [Holótipo macho. Pico da Tijuca, Rio de Janeiro, Brasil].

Ref.: Amedegnato \& Descamps (1979: 449).

\section{Seabracris Amedegnato \& Descamps 1979: 462.}

tijuca Amedegnato \& Descamps 1979: 466.

Distribuição geográfica: Brasil (RJ).

Tipo: MNRJ [Holótipo macho. Fl. da Tijuca, Rio de Janeiro, Brasil].

Ref.: Amedegnato \& Descamps (1979: 466).

\section{Caloscirtae}

Abracrini

\section{Abracris Walker, 1870: 642}

Osmilia Stal, 1873: 68

*dilecta Walker, 1870: 642.

caeruleipennis Bruner, 1900: 68

conspersipennis Bruner, 1908: 281

meridionalis Bruner, 1908: 281

signatipes Bruner, 1906: 673

Distribuição geográfica: México, América Central, América do Sul, da Colômbia ao Uruguai; Brasil (PA, BA, GO, MT, MG, RJ, RS).

Tipo: BMNH [Holótipo macho. Brasil, Santarém].

Refs.: WALKer (1870: 642); BRUNER (1900: 68, 1906: 673); REHN (1913: 273, 1915: 270, 1916: 294, 1918: 183); LIEBERMANN (1928: 137, 1939: 125, 1945, 235, 1951: 39, 1958: 3, 1967: 96); HEPPER (1945: 280); CARBONELl et al. (1980: 280); RoBERTS \& CARBONELL (1981: 3); SPerber (1996: 127); CARBonell et al. (2007: 57); BUZETTI \& CAROTTI (2008: 57).

\section{flavolineata (De Geer), 1773: 497. (Acrydium)}

coelestre Burmeister, 1839: 634

labrata Scudder, 186: 634

saussurei Scudder, 1875: 274

tolteca Saussure, 1861: 163

violacea Thunberg, 1824: 413

Distribuição geográfica: Santa Lucia (Windward Islands); Tobago; México; América Central; América do Sul, da Colômbia até metade da Argentina e Brasil (PA, RO, AM, AP, BA, PE, RN, RJ, RS).

Tipo: perdido [Suriname].

Refs.: De GeER (1773: 497); BRUNER (1900: 80, 1908: 331, 1919: 88); REHN (1913: 273, 1915: 270, 1916: 295, 1918: 184); HEBARD (1923: 265, 1924a: 189, 1924b: 131, 1925: 283, 1932: 274, 1933a: 130, 1933b: 46); LiEBERMANN (1928: 137, 1939: 125, 1945: 235, 1958: 3); ROBERTS (1937: 356); HEPPER (1945: 280); HAYWARD (1960: 3); Descamps \& Amedegnato (1970: 892); Descamps (1975: 32); GANGwERE \& Ronderos (1975: 173); Rowell (1978: 653, 1983: 750); TURK (1980: 122, 1981: 53); CARBONELl et al. (1980: 280); RoBERTS \& CARBONELl (1981: 3; C.O.P.R. (1982: 195); Flook \& Rowell (1997: 89); CARBONELl et al.( 2007:57); Fontana et al. (2008: 206); Buzetti \& CARotti (2008: 57); BARRANCO (2010: 28); CARDONA (2012: 70); RowELL \& BEHRSTOCK (2012: 242); BARRIENTOS LozANo et al. (2013: 319).

\section{Sitalces Stål 1878: 16}

*volxemi Stål 1878: 16

Distribuição geográfica: Brasil (RJ).

Tipo: NHRS [Rio de Janeiro, Brasil].

Refs.: STÅL (1878: 16); Kirby (1910: 435); MatiotTi dA Costa \& Silva Carvalho (2006: 139); Matiotti da Costa et al. (2007: $383)$. 
borellii Giglio Tos 1898: 48

Distribuição geográfica: Brasil (MS, MT, SP, RJ); Paraguai (Paraguari).

Tipo: MIZT [Paraguai, Sapucai].

Refs.: Giglio-Tos (1898: 48, 1900: 5); REHN (1907: 186); BRUNER (1919: 77); Passerin d'Entrèves (1981: 49); Descamps (1979: $313)$.

\section{(Ommatolampidinae incertae sedis)}

\section{Beckeracris Amedegnato \& Descamps 1979: 471.}

janeirensis Amedegnato \& Descamps 1979: 476.

Distribuição geográfica: Brasil (RJ).

Tipo: MNRJ [Holótipo macho. Fl. da Tijuca, Rio de Janeiro, Brasil].

Ref.: Amedegnato \& Descamps (1979: 476).

\section{Cyrtacanthacridinae}

Cyrtacanthacridini

\section{Schistocerca Stål 1873: 64}

flavofasciata (De Geer) 1773: 498 (Acrydium)

aequalis Scudder, 1899: 444

desiliens Scudder, 1899: 443

femorale Walker, 1870: 620

fimbriatus Thunberg, 1824: 400

flavolinea Bruner, 1920: 19

infumata Scudder, 1899: 444

longipenne Burmeister, 1838: 632

parvula Walker, 1870: 567

septentrionalis Walker, 1870: 555

subvittata Walker, 1870: 620

vittatum Olivier, 1791: 221

Distribuição geográfica: Suriname; Guiana; Guiana Francesa; Curaçao (Dutch West Indies); Trinidad; N.Venezuela; Brasil (PA, AM, MT, RN, PB, PE, SE, BA, ES, MG, RJ, SP, PR, SC, RS); (?) Peru (Junin, Puno); Bolívia (?) (La Paz, Santa Cruz); L.Paraguai; Argentina (Misiones, Corrientes, Entre Rios, Formosa, Chaco, Santa Fé, Cordoba, Buenos Aires); Uruguai.

Tipo: BMNH [Holótipo fêmea. Sierra de la Unrosa, Riviera, Uruguai].

Refs.: De Geer (1773: 489); Olivier (1791: 221); Thunberg (1824: 428); WALKER (1870: 552); SCUDDER (1899: 455); KIRBY (1910: 456); REHN (1916: 304); BRUNER (1919: 82); BRUCH (1939: 209); LiEBERMANN (1939: 125, 1940: 155, 1945: 235, 1948: 55, 1951: 39, 1954); Daguerre (1940: 327); Dirsh (974: 157); BARRERA \& Paganini (1975: 107); C.O.P.R. (1982: 316); Cigliano \& Lange (1998: 129); Song (2004: 420); CARBOnELl et al. (2006: CD Rom); De Souza \& Melo (2007: 54).

nitens nitens (Thunberg) 1815: 236 (Gryllus)

aurantia Scudder, 1899: 443

australis Scudder, 1899: 444

bogotensis Scudder, 1899: 444

boyacae Hebard,

carinata Scudder, 1899: 443

concolor Walker, 1870: 650

consobrinum Walker, 1870: 636

crococtaria Scudder, 1899: 443

gracilis Scudder, 1899: 443

gulosa Scudder, 1899: 444

implecta Walker, 1870: 554

lividus Thunberg, 1824: 102

luridescens Walker, 1870: 583

malachitica Rehn, 1905: 439

maya Scudder, 1899: 444

mellea Scudder, 1899: 443

occidentale Scudder, 1875: 274

perturbans Scudder, 1899: 446

proprium Walker, 1870: 621

pyramidata Scudder, 1899: 443

scutellare Walker, 1870: 579

semivittatum Walker, 1870: 583

separata Scudder, 1899: 446

sonorensis Scudder, 1899: 445

strenuum Walker, 1870: 234

vaga Scudder, 1876: 269

vitticeps Walker, 1870: 579

zapoteca Scudder 1899: 443

Distribuição geográfica: México (Oaxaca, whole country); USA (California, Arizona, New México); Guatemala; Belize; Honduras; El Salvador; Nicaragua; Costa Rica; Panamá; Bahamas; Trinidad; Tobago; Curaçao; Bonaire; Equador; Colômbia; Venezuela; Guiana; Suriname; Guiana Francesa; Brasil (RR, PA, AM, MT, MS, MA, CE, RN, PE, AL, BA, GO, ES, RJ, PR, SC, RS); L.Paraguai; Argentina (La Rioja, San Juan); Chile (Tarapaca, Antofagasta).

Tipo: UZIU [Holótipo macho. "North America"].

Refs.: THunBerg (1815: 236); STÅL (1873: 67); Dirsh (1965: 38, 1974: 94); DESCAMPS (1975: 58); C.O.P.R. (1982: 317); VICKERY \& Kevan (1983: 720); Richman et al. (1993: 91); Elgueta et al. (1999: 34); CAPINERA et al. (2004: 146); CARBONELL et al. (2006: CD Rom); Rivera García (2006: 142); CARBONELl et al. (2007: 57); BuzetTI \& CAROtTI (2008: 58); LockWOOd \& LATCHININSKY (2008: 429); BARRANCO (2010: 27); CARDONA (2012: 71); BARrientos LozANo et al. (2013: 319); Rowell (2013: 193). 


\section{Acridinae}

Eutryxalis Bruner 1900: 22, 24

Paratruxalis Bruner 1900

filata filata (Walker) 1870: 785 (Chrysochraon)

Distribuição geográfica: Brasil (PA, MG, RJ, SP, SC, RS, MT, MS); Trinidad; Paraguai (Concepcion, Paraguari, Guaira, Itapua); Argentina (Misiones).

Tipo: BMNH [Holótipo macho. Santarém, Pará, Brasil].

Refs.: WALKER (1870: 785); REHN (1916: 275, 1944: 209); BRUNER (1919[1920]: 25); HEBARD (1923: 197, 1931: 270); LiEBERMANN (1939a: 150); JAGO (1971: 213); DONATO (2003: 392); CARBONELL et al. (2006: CD Rom).

\section{Orphula Stål 1873: 94, 105}

Sisantum, Bruner, 1902: 30

Thyriptilon, Bruner, 1902: 30

crassa (Bruner) 1911: 17 (Orphulella)

Distribuição geográfica: BRASIL Rio de Janeiro (provável sinonímia de pagana)

Tipo: Não localizados [Sintipos Macho e Fêmea. Rio de Janeiro, Brasil].

Refs.: BRUNer (1911: 17); OTTE (1979: 56).

*pagana pagana (Stål) 1860: 339 (Gomphocerus)

Distribuição Geográfica: Brasil (RJ).

Tipo: NHRS [Holótipo macho. Rio de Janeiro, Brasil].

Refs.: STÅL (1861: 339, 1873: 106); BRUNER (1900: 23); REHN (1906: 16); GANGWERE \& Ronderos (1975: 173); CARBonell et al. (2006: CD Rom).

Gomphocerinae

\section{Compsacrini}

\section{Silvitettix Bruner 1904: 55}

Charpentieracris Descamps \& Amedegnato, 1970: 893

Leuconotus Bruner, 1904: 57

Ochrotettix Bruner, 1900-09: 56

Oaxacella Hebard, 1932: 231

territus Otte \& Jago 1979: 280

Distribuição geográfica: Brasil (MG, RJ).

Tipo: MNRJ [Holótipo macho. Poços de Caldas, Minas Gerais, Brasil].

Refs.: Otte \& JaGo (1979: 280); De Vasconcellos \& Monné (2001: 77)

\section{Staurorhectus Giglio-Tos, 1897: 25}

longicornis longicornis Giglio-Tos, 1897: 26

Distribuição geográfica: Brasil (BA, GO, MT, MG, RJ, SP, RS); Uruguai (Cerro Largo); Paraguai (Caaguazu).

Tipo: MIZT [Argentina (sem localidade exata)].
Refs.: Giglio-Tos (1897: 26); Rehn (1906a: 33, 1906b: 376); Kirby (1910: 106); ReHN (1913: 317); Bruner (1919: 31); HEBARD (1931: 271); Roberts (1937: 349); LiEBERMANN (1939: 157); PAsserin D'Entreves (1981: 45); C.O.P.R. (1982: 555); Riede (1987: 271); BENTOS-PEREIRA \& LoRIER (1991: 631); LANGE (1992: 65); SÁnchez \& de Wysiecki (1993: 65); Cigliano \& Lange (1998: 67); Cigliano et al. (2000: 81); Cigliano et al. (2002: 215); DE WySIECKi et al. (2004: 87); CARBONELL et al. (2006: CD Rom); MARIOTTINI et al. (2011: 737).

\section{Gomphocerini}

\section{Stauroderus Bolívar, 1897: 224}

campestris (Stål) 1860: 341 (Stenobothrus) [uma espécie do Rio de Janeiro, que Kirby (1910) classifica como Stauroderus. Status incerto, AMÉDÉGNATO \& CARBONELL (2001) em "List of Neotropical genera of acridomorph Caelifera”]

Distribuição geográfica: Brasil (RJ).

Tipo: NHRS [Holótipo macho. Rio de Janeiro, Brasil].

Ref.: STÅL (1860: 341); KiRBY (1910: 183).

\section{Orphulellini}

\section{Orphulella Giglio-Tos, 1894: 10}

Isonyx Rehn, 1906: 36

Linoceratium Bruner, 1904: 84

Parachloebata Bruner, 1904: 83

punctata (De Geer), 1773: 503. (Acrydium)

arctatus Walker, 1870: 761

compacta Bruner, 1911: 19

costalis Walker, 1870: 759

costaricensis Bruner, 1904: 82

elegans Giglio-Tos, 1894: 12

elongata Bruner, 1911: 14

expandens Walker, 1870: 758

gracilis Giglio-Tos, 1894: 11

gratiosus Walker, 1870: 759

grossa Bruner, 1911: 18

insularis Bruner, 1906: 150

interrupta Bruner, 1911: 12

intricata Stal, 1873: 106

meridionalis Bruner, 1904: 81

mexicanus Walker, 1870: 756

tepanecus Saussure, 1861: 319

totonacus Saussure, 1861: 315

virdissimus Walker, 1870: 760

zapotecus Saussure, 1861: 316

Distribuição geográfica: México; Cuba; Hispaniola; Porto Rico; América Central; América do Sul, Brasil (RJ). 
Tipo: NHRS [Lectotipo fêmea. Suriname].

Refs.: De Geer (1773: 503); Brunner von Wattenwyl \& Redtenbacher (1892: 208); MCNeIll (1897: 235); PrinZEssin VON BAYERN (1900: 255); REHN (1903: 133, 1905a: 802, 1905b: 401, 1905c: 177, 1906: 373, 1913: 316, 1916: 277, 1918: 169); KIRBY (1910: 121); BRUNER (1910: 468, 1919: 26); HEBARD (1923: 207, 1924: 172, 1933a: 128, 1933b: 44); UvAROv (1925: 269); ROBERTS (1937: 349); Piza JR. (1945: 184); Descamps \& AmÉdÉgnato (1970: 895); ОтTE (1979: 52); ОтTе (1981: 243); AMEDEGNATO et al. (1995: 702); CARBONELl et al. (2006: CD Rom); CARBONELL et al. (2007: 59); FonTANA et al. (2008: 149); BuzETTI \& CAROTTI (2008: 60); BARRANCO (2010: 27); MARIOTTINI et al. (2011: 737); CARdona (2012: 73); Barrientos Lozano et al. (2013: 198); Rowell (2013: 236).

\section{Orphulina Giglio-Tos 1894: 9}

\section{*pulchella Giglio-Tos 1894: 9}

Distribuição geográfica: Paraguai (San Pedro, Paraguari, Guairá); Guiana Francesa; Panamá (Darien); Colômbia (Vaupes, Meta, Amazonas); Brasil (RJ, ES, MT, BA); Bolívia (Santa Cruz).

Tipo: MIZT [Holótipo macho. Bovince, San Pedro, Paraguai].

Ref.: Giglio-Tos (1894: 8, 1897: 2); Kirby (1910: 118); Hebard (1924: 94); LiEBERMANN (1939: 125); ОTTE (1979: 52, 1981: 274); Passerin d'Entreves (1981: 34); Carbonell et al. (2006: CD Rom); CARBonell et al. (2007: 59); Rowell (2013: 244).

\section{Scyllinini}

\section{Rhammatocerus Saussure, 1860}

pseudocyanipes Assis-Pujol, 1997:4

Distribuição geográfica: Brasil.

Tipo: MNRJ [Holótipo macho. Brasil, Sergipe, Areia Branca].

Refs.: Assis-Pujol (1997: 4); Buys (2009:311); Guerra et al. (2012:5).

victori Dos Santos \& Assis-Pujol, 2003:171

Distribuição geográfica: Brasil (RJ).

Tipo: MNRJ [Holótipo macho. Brasil, Rio de Janeiro, Barra da Tijuca].

Ref: Alves dos Santos \& Assis-Pujol (2003:171).

\section{EUMASTACOIDEA}

\section{PROSCOPIIDAE}

\section{Proscopiinae}

\section{Corynorhynchus Brunner von Wattenwyl, 1890:101}

\section{childei Mello Leitão 1939: 344}

Distribuição geográfica: Brasil (RJ).

Tipo: perdido [Brasil, RJ].

Refs.: Mello-Leitão (1939: 344); Carbonell (1977: 17); PiZa JR. (1979: 86); LiANA (1980: 245).

radula (Klug), 1820: 20 (Proscopia)

ruficornis Klug

Distribuição geográfica: Brasil (RJ).
Tipo: ZMHB [Lectótipo fêmea. "Rio v. Olf”].

Refs.: KLUG (1820: 20); Burmeister (1838: 604); BRUNNER VON WaltTENWYL (1890: 102); ReHN (1904: 677); KirBy (1910: 85); Bruner (1913: 433); HeBARD (1924: 163); MELlo-LeITÃo (1939: 350); Carbonell (1977: 18); Aguilar (1983: 87); JaGo (1989: 273).

\section{AGRADECIMENTOS}

Os autores agradecem a A.M. Bentos-Pereira, M.G. Lhano e R.S. Olivier, pelo envio de artigos científicos. Agradecemos também ao CNPq e FAP-DF, pelo auxílio concedido (JRPL).

\section{REFERÊNCIAS}

Adis, J., E. Bustorf, M.G. Lhano, C. Amedegnato \& A.L. Nunes, 2007. Distribution of Cornops grasshoppers (Leptysminae: Acrididae: Orthoptera) in Latin America and the Caribbean Islands. Studies on Neotropical Fauna and Environment, 42: 11-24.

Adis, J. \& W.J. Junk, 2003. Feeding impact and bionomics of the grasshopper Cornops aquaticum on the water hyacinth Eichhornia crassipes in Central Amazonian floodplains. Studies on Neotropical Fauna and Environment, 38: 245249.

Adis, J., M.G. Lhano, M.P. Hill, W.J. Junk, M.I. Marques \& H. Oberholzer, 2004. What determines the number of juvenile instars in the tropical grasshopper Cornops aquaticum (Leptysminae: Acrididae: Orthoptera). Studies on Neotropical Fauna and Environment, 39: 127-132.

Aguilar, F., 1983. Especies de proscópidos registrados para el Perú. Revista Peruana de Entomología, 26: 85-88.

Alves dos Santos, C.M. \& C.V. Assis-Pujol, 2003. Espécie nova de Rhammatocerus Saussure, 1861 do Estado do Rio de Janeiro, Brasil (Caelifera, Acrididae, Gomphocerinae, Scyllinini). Arquivos do Museu Nacional, 61: 171-174.

Amédégnato, C. \& M. Descamps, 1979. Diagnoses génériques et affinités phylétiques d'Acridoidea néotropicaux récoltés par le Dr. Campos Seabra et M.Descamps (Orthoptera). Annales de la Societé Entomologique de France (NS), 15: 423-487.

Amédégnato, C. \& M. Descamps, 1982. Dispersal centers of the Amazonian acridids. Acta Amazônica, 12: 155-165.

Amedegnato, C., A. Ruiz-Baliú \& C.S. Carbonell, 1995. Acridofauna Cubana (Orthoptera): sinopsis de su taxonomia e origen. Revista Brasileira de Entomologia, 39: 683-708.

Andres, L.A. \& F.D. Bennett, 1975. Biological control of aquatic weeds. Annual Review of Entomology, 20: 31-46.

Araújo Cisneiros, R., A. Vasconcelos de Almeida, G. Rivas de Melo \& C.A. Gomes da Câmara, 2012., Morphometric variations in the grasshopper, Chromacris speciosa from two localities of pernambuco in northeastern Brazil. Journal of Insect Science, 12: 79 (10p).

Assis-Pujol, C.V.A., 1997. Duas novas espécies brasileiras de Rhammatocerus Saussure, 1861 (Acrididae, Gomphocerinae, Scyllinini). Boletim do Museu Nacional: nova série Zoologia, 380: 1-10.

Barranco, P., 2010. Ortópteros de la Reserva Biológica Alberto Manuel Brenes (Costa Rica) II. Orthoptera: Caelifera. Boletín de la Sociedad Entomológica Aragonesa, 47: 21-32.

Barrera, M. \& I.H. Paganini, 1975. Acridios de Tucumán: notas bioecológicas. Acta Zoológica Lilloana, 31: 107-124.

Barrera, M. \& S.Z. Turk, 1977. Acridios del NOA, II. Contribución al conocimiento de huevos, desoves y hábitos de postura de algunas especies de tucuras (Orthoptera, Acrididae), de la Provincia de Tucumán. Acta Zoológica Lilloana, 32: 167-188.

Barrientos Lozano, L., A.Y. Rocha-Sánchez, F.M. Buzzetti, B.L. Méndez-Gómez \& J.V. Horta-Vega, 2013. Saltamontes y esperanzas del noreste de México (Insecta: Orthoptera). Guía ilustrada, 388p.

Beingolea Guerrero, O., 1989. Lista de acrídidos (OrthopteraCaelifera: Acrididae), registrados para el Perú. Revista 
Peruana de Entomología, 32: 37-40.

Bennett, F.D., 1970. Insects attacking water hyacinth in the West Indies, British Honduras and the U.S.A. Hyacinth Control Journal, 8: 10-13.

Bennett, F.D. \& H. Zwolfer, 1968. Exploration for natural enemies of the water hyacinth in northern South America and Trinidad. Hyacinth Control Journal, 7: 44-52.

Bentos-Pereira, A., 1989. Distribución geográfica de las especies del género Dichroplus Stål (Orthoptera, Acrididae, Melanoplinae). Revista Brasileira de Entomologia, 33: 31-47

Bentos-Pereira, A. \& E. Lorier, 1991. Acridomorfos acuáticos (Orthoptera, Acridoidea) I. Adaptaciones morfológicas. Revista Brasileira de Zoologia, 35: 631-653.

Berg, C., 1881. Entomologisches aus dem Indianergebiet der Pampa. Stettiner Entomologische Zeitung, 42: 36-72.

Bidau, C.J., 2012. Curious orthopterological errors of a great naturalist: William Henry Hudson. Journal of Orthoptera Research, 21: 255-260.

Blanchard, E., 1836. Monographie du genre Ommexecha de la famille des Acridiens. Annales de la Société Entomologique de France, 5: 603-624.

Blanchard, E., 1945. Insectos y nematodos relacionados con el cultivo del tabaco. Ministerio de Agricultura, Departamento de Sanidad Vegetal, Santiago. 1: 23p.

Bolivar, C., 1884. Insectos Neurópteros y Ortópteros. p. 30-51, 1 am. 1-3. In: Artrópodos del Viaje al Pacífico, verificado de 1862 a 1865, por una comisión de Naturalistas enviada por el Gobierno Español. Madrid, 115p.

Bolívar, I., 1888. Enumération des Orthoptères de l'île de Cuba. Mémoires de la Société Zoologique de France, 1: 116-164.

Bolívar, I., 1890. Diagnosis de orthópteros nuevos. Annales de la Sociedad Española de Historia Natural, 19: 299-334.

Bolívar, I., 1896. Ortópteros recogidos por el Sr. Uraz en la cuena del Rio Atalapo (misprint for Atabapo). Annales de la Sociedad Española de Historia Natural, Ser. 2, 5: 11-18.

Bolívar. I., 1906. Rectificaciones y observaciones ortopterológicas. Boletin de la Real Sociedad Española de Historia Natural, 6: 387-396.

Bolívar, I., 1909. Acridios nuevos americanos. Revista Chilena de História Natural, 13: 337-354.

Braga, C.E., J.W. Nunes \& J. Adis, 2007. Cornops frenatum frenatum (Marschall, 1836) (Orthoptera, Acrididae, Leptysminae): Ocorrência e oviposição em quatro espécies de Heliconia (Heliconiaceae) na Amazônia Central, Brasil. Amazoniana, 19: 227-231.

Braga, C.E., AL. Nunes-Gutjahr, J.W. Morais \& J. Adis, 2011. Fenologia de Cornops aquaticum (Orthoptera: Acrididae) associado a Eichhornia crassipes (Pontederiaceae) em um Lago de Várzea na Amazônia Central, Brasil. Revista de la Sociedad Entomológica Argentina, 70: 185-196.

Braker, H.E., 1989. Evolution and ecology of oviposition on host plants by acridoid grasshoppers. Biological Journal of the Linnean Society, 38: 389-406.

Bruch, C., 1939. Algunas observaciones biológicas sobre Schistocerca infumata Scudd. (Acrid.). Revista del Museo de La Plata (1891-1934), 1: 209-216.

Bruner, L., 1895. Nicaraguan Orthoptera. Bulletin from the laboratories of natural history of the State University of Iowa, 3: 58-69.

Bruner, L., 1900-1909. Acrididae. In: Biologia Centrali Americana, 2: 1-342, pl., 1-4.1904, 33-104p; 1906, 177-208; 1908, 249-342p.

Bruner, L., 1900. The second report of the Merchant's Locust Inveigation Commission of Buenos Aires. Lincoln, Nebraska, 8op.

Bruner, L., 1906. Synoptic list of Paraguayan Acrididae or Locusts, with descriptions of new forms. Proceedings of the United States National Museum, 30: 613-694.

Bruner, L., 1908. Orthoptera. The Acrididae. Biologia CentraliAmericana, 2: 249-342.

Bruner, L., 1910. Report on an interesting collection of locusts from Peru. Horae Societatis Entomologicae Rossicae, 39: 464-488.

Bruner, L., 1911. I.South American Acridoidea. Annals of the Carnegie Museum, 8: 5-147.

Bruner, L., 1913. South American Locusts (Acridoidea). II. Annals of the Carnegie Museum, 8: 423-506.

Bruner, L., 1919. I. Saltatorial Orthoptera from South America and the Isle of Pines. Annals of the Carnegie Museum, 13: 5-91.

Bruner von Wattenwyl, K., 1890. Monographie der Proscopiden. Verhandlugen der k. k. zoologisch-botaniscen Gesellschaft in Wien, 40: 87-124.

Brunner von Wattenwyl, C. \& J. Redtenbacher, 1892. On the Orthoptera of the Island of St. Vincent, West Indies. Proceedings of the Zoological Society of London. 196-220, Pl. 15-17.

Burmeister, H., 1838. Handbuch der Entomologie. Zweiter Band. Berlin, 1050p.

Buys, S.C., 2009. Reproductive behaviour and larval development of Prionyx thomae (Fabricius) from Brazil. Journal Beiträge zur Entomologie, 59: 311-317.

Buzzetti, F.M. \& Carotti, G., 2008. Annotated list of the Caelifera of Ecuador (Biodiversity of South America). Memoirs on Biodiversity, 1: 39-66.

Campodonico, M.J. \& F.H. Santoro, 1971. Identificación de acridios (Orthoptera) por las esculturas del corion. Revista de Investigaciones Agropecuarias, VIII: 63-82.

Capello, S., J. Adis \& M.L. Wysiecki, 2007. Temperatura y fotoperíodo: qué influencia ejercen en el desarrollo ninfal de Cornops aquaticum (Orthoptera: Acrididae)? Amazoniana, XIX: 209-216.

Capello, S., M. Marches \& M.L. Wysiecki, 2012. Feeding Habits and Trophic Niche Overlap of Aquatic Orthoptera Associated with Macrophytes. Zoological Studies, 51: 51-58.

Capello, S., M.L. Wysiecki \& M. Marchese, 2011. Feeding patterns of the aquatic grasshopper Cornops aquaticum (Bruner) (Orthoptera: Acrididae) in the middle Paraná river, Argentina. Neotropical Entomology, 40: 170-175.

Capinera, J.L, R.D. Scott \& T.J. Walker, 2004. Field Guide to Grasshoppers, Katydids, and Crickets of the United States. 249.

Carbonell, C.S., 1969. Revision of the genus Zygoclistron Rehn, 1905 (Orthoptera, Acridoidea). Transactions of the American Entomological Society, 95: 571-602.

Carbonell, C.S., 1977. Superfam. Proscopioidea, fam. Proscopiidae. Pars 17 of Max Beier (ed.) Orthopterorum Catalogus. W.J.Junk, the Hague, 29p.

Carbonell, C.S., 1981. Orthoptera, p. 92-99. In: Hurlbert, S.H., G. Rodriquez \& N.D. Santos (Eds.). Aquatic biota of tropical South America. Part 1, Arthropoda, 342p.

Carbonell, C.S., 1984. Nomenclature and systematics of Tropidacris and Eutropidacris (Orthoptera: Acridoidea, Romaleidae). Notulae Naturae, 461: 1-11.

Carbonell, C.S., 1986. Revision of the neotropical genus Tropidacris (Orthoptera, Acridoidea, Romaleidae, Romaleinae). Proceedings of the Academy of Natural Sciences of Philadelphia, 138: 366-402.

Carbonell, C.S., 1989. Digamacris n. gen. (Orthoptera, Acrididae, Melanoplinae) de la region Atlântica meridional de Brasil. Memórias do Instituto Oswaldo Cruz, 84: 77-86.

Carbonell, C.S., 1995. Revision of the tribe Scyllinini, nov. (Acrididae: Gomphocerinae), with descriptions of new genera and speces. Transactions of the American Entomological Society, 121: 87-152.

Carbonell, C.S. \& R.A. Ronderos, 1973. Las espécies del grupo "punctulatus" del género Dichroplus Stal. Revista del Museo de La Plata (N.S.) Zoologia, 11: 359-398.

Carbonell, C.S., A. Mesa \& L. Condutta, 1980. Systematics and Kariotype of the grasshopper genera Abracris and Ommalotettix. Proceedings of the Academy of Natural Sciences of Philadelphia, 132: 279-284. 
Carbonell, C.S., M.M. Cigliano \& C.E. Lange, 2006. Especies de acridomorfos (Orthoptera) de Argentina y Uruguay. Publicacion especial bilingue (castellano / ingles) en CD financiada por la «Orthopterists Society», USA y editada por el Museo de la Plata.

Carbonell, C.S., C.H.F. Rowell, A. Bentos-Pereira \& M.F. Porras, 2007. Checklist of Orthoptera Caelifera from Colombia. Zootaxa, 1594: 39-59.

Cardona, J.M., 2012. Grasshoppers of Northwest South America. A Photo Guide. Volume 1 - The Western Fauna, 124p.

Carl, J., 1916. Acridides nouveaux ou peu connus du Museum de Geneve. Revue Suisse de Zoologie, 24: 461-518.

Carvalho, D.B., M. Rocha, V. Loreto, Silva \& M.J. de Souza, 2011. Ommexcha vires (Thunberg, 1824) and Descampacris serrlatum (Serville, 1831) (Orthoptera, Ommexechidae): karyotypes, constitutive heterochromatin and nucleous organizing regions. Comparative Cytogenetics, 5: 123-132

Castillo, E.R.D., Bidau \& D.A. Marti, 2010. Neo-sex chromosome diversity in Neotropical melanoplinae grasshoppers (Melanoplinae, Acrididae). Genetica, 138: 775-786.

Chapco, W., 2006. A note on the phylogenetic position of Duartettix montanus within the subfamily Melanoplinae. Journal of Orthoptera Research, 15: 59-63.

Cigilano, M.M., 1997. Ronderosia, a new genus of South American Melanoplinae (Orthoptera, Acridoidea). Journal of Orthoptera Research, 6: 1-19.

Cigliano, M.M., M.L. deWysiecki \&C.E. Lange, 2000. Grasshopper (Orthoptera: Acridoidea) species diversity in the Pampas, Argentina. Diversity and Distributions, 6: 81-91.

Cigliano, M.M. \& R.A. Ronderos, 1994. Revision of the South American Grasshopper Genera Leiotettix Bruner and Scotussa Giglio-Tos (Orthoptera, Acrididae, Melanoplinae). Transactions of the American Entomological Society, 120: 145-180.

Cigliano, M.M. \& C.E. Lange, 1998. Orthoptera, p. 67-83 In: Morrone, J.J. \& S. Coscaron. Biodiversidad de Artrópodos Argentinos: Una Perspectiva Biotaxonómica, 599p.

Cigliano, M.M., S. Torrusio \& M.L. deWysieck, 2002. Grasshopper (Orthoptera: Acrididae) community composition and temporal variation in the Pampas, Argentina. Journal of Orthoptera Research, 11: 215-221.

Cigliano, M.M., M.L. de Wysiecki \& C.E. Lange, 1995. Disminucion de la abundancia de Dichroplus maculipennis (Orthoptera: Acridoidea) en comunidades del sudoeste de la Provincia de Buenos Aires, Argentina. Revista de la Sociedad Entomológica Argentina, 54: 41-42.

Cigliano, M.M., R. Ronderos \& W.P. Kemp, 1996. Phylogenetic relationships of Scotussa and Leiotettix (Orthoptera: Acrididae). Cladistics, 12: 125-138.

Cigliano, M.M., M.L. deWysiecki \& C.E. Lange. 2000. Grasshopper (Orthoptera: Acridoidea) species diversity in the Pampas, Argentina. Diversity and Distributions, 6: 81-91.

Cigliano, M.M., S. Torrusio \& M.L. de Wysiecki, 2002. Grasshopper(Orthoptera:Acrididae) community composition and temporal variation in the Pampas, Argentina. Journal of Orthoptera Research, 11: 215-221.

Cilliers, C.J., 1991. Biological control of water fern, Salvinia molesta (Sal-viniaceae), in South Africa. Agriculture, Ecosystems \& Environment, 37: 219-224.

Colombo, P.C., M.M. Cigliano, A.S. Sequeira, C.E. Lange, J.C. Vilardi \& V.A. Confalonieri, 2005. Phylogenetic relationaships in Dichroplus Stål (Orthoptera: Acrididae: Melanoplinae) inferred from molecular and morphological data: testing karyotype diversification. Cladistics, 21: 375-389.

C.O.P.R., 1982. The Locust and grasshopper agricultural manual Published by the Centre for Overseas Pest Research, London, vii $+690 p$.

Daguerre, J.B., 1940. El genero Schistocerca en la Republica Argentina. Revista de la Sociedad Entomológica Argentina, 10: 327-337.

Da Silva, F.R.J., M.I. Marques, L.D. Battirolla \& M.G. Lhano, 2010.
Variação do peso fresco em Cornops aquaticum (Bruner) (Orthoptera, Acrididae) associado a Eichhornia azurea (Sw) Kunth (Pontederiaceae) em uma baía no Pantanal de Poconé, Mato Grosso. Neotropical Entomology, 39: 535-542.

De Geer, C., 1773. Onziéme memoire, Des criquets, p. 460-504, In: Memoires pour servir a l'histoire des insectes. Stockholm, Tome 3, viii + $697 \mathrm{p}$.

de Paulo Lemos, W., R.C. Ribeiro, M.G. Lhano; J.P. Santos da Silva \& J.C. Zanuncio, 2010. Cornops frenatum frenatum (Marschall) (Orthoptera: Acrididae, Leptysminae) in crops of tropical flowers of Heliconia spp. in the State of Pará, Brazil. Entomotropica, 25: 43-47.

de Souza, M.J. \& N. F. de Melo, 2007. Chromosome study in Schistocerca (Orthoptera-Acrididae-Cyrtacanthacridinae): karyotypes and distribution patterns of constitutive heterochromatin and nucleolus organizer regions (NORs). Genetics and Molecular Biology, 30: 54-59.

Descamps, M., 1975. Etude du peuplement acridien de l'état de Veracruz (Mexique). Folia Entomologica Mexicana, 31-32: 3-97.

Descamps, M., 1976. La faune dendrophile néotropicale. I. Revue des Proctolabinae (Orthoptera, Acrididae), Acrida, 5: 63-167.

Descamps, M., 1977. Notes d'acridologie neotropicale, 1. (Orth.). Bulletin de la Société Entomologique de France, 82: 63-77.

Descamps, M., 1979. Missions entomologiques en Guyane et au Brésil. Trosiéme note: Orthoptera Acridomorpha. Annales de la Société Entomologique de France (MN), 15: 311-317.

Descamps, M., 1980. La faune dendrophile néotropicale. V. Seconde revue des Proctoclabinae amazoniens et guyanais (Orthoptéres, Acrididae).Annales dela Société Entomologique de France (N.S.), 16: 19-47 e 161-195.

Descamps, M., 1983. Notes sur les types d'Éumastacoidea et d'Acridoidea décrits par M. Perty. Spixiana, 9: 291-294 (Supplement).

Descamps, M., 1984. Revuepréliminaire dela tribu des Copiocerini (Orth. Acrididae). Mémoires du Muséum National d'Histoire Naturelle. Ser. A, Zool, 130: 1-72.

Descamps, M. \& C. Amedegnato, 1970. Acridomorpha (Orthoptera) rácoltés em Guyane Française par la mission du Museum National d"Histoire Naturelle. Annales de la Société entomologique de France (N.S.), 6: 861-897.

Descamps, M. \& C. Amedegnato, 1972. Contribution a la faune des Acridoidae de Colombie (Missions M. Descamps). III. Diagnoses de Catantopinae (sensu lato). Annales de la Société Entomologique de France (N.S.), 8: 505-559.

Descamps, M. \& C. Amedegnato, 1989. Les genres Vilerna, Locheuma et Pseudovilerna nov. I Le genre Vilerna Stal, 1873 (Orthoptera, Acrididae, Ommatolampinae). Revue Française d'Entomologie (N.S.), 11: 17-28.

Descamps, M. \& C.S. Carbonell, 1985. Revision of the neotropical arboreal genus Titanacris (Orthoptera, Acridoidea, Romaleidae). Annales de la Société Entomologique de France (N.S.), 21: 259-285.

de Vasconcellos, S.M. \& M. A. Monné, 2001. Tipos primários de Orthoptera (Caelifera) da coleção do Museu Nacional, Rio de Janeiro. Publiçacões Avulsas do Museu Nacional, 87: 19p.

de Wysiecki, M.L., S. Torrusio \& M.M. Cigliano, 2004. Caracterización de las comunidades de acridios (Orthoptera: Acridoidea) del partido de Benito Juárez, sudeste de la provincia de Buenos Aires, Argentina. Revista de la Sociedad Entomológica Argentina, 63: 87-96.

Dias-Guerra, W., P.C. De Oliveira \& L. Barrientos Lozano, 2010. Life history and population dynamics of Baeacris punctulatus (Thunberg, 1824) (Orthoptera: Acrididae) in the state of Mato Grosso, Brazil. Journal of Orthoptera Research, 19: 333-340.

Dirsh, V.M., 1956. The phallic complex in Acridoidae (Orthoptera) in relation to taxonomy. Transactions of the Royal Entomological Society of London, 108: 223-356.

Dirsh, V.M., 1965. Preliminary note for the revision of the genus Schistocerca Stål, 1873 (Orthoptera, Acridoidea). EOS, 41: 31-43. 
Dirsh, V.M., 1974. Genus Schistocerca (Acridomorpha, Insecta). Series Entomologica, vol. 10, W. Junk, The Hague, vii +238 pp.

Donato, M., 2000. Los ejemplares tipo de Orthoptera depositados en la colección del Museo de La Plata. Revista de la Sociedad Entomológica Argentina, 59: 61-84.

Donato, M., 2003. Revision of the South American Genera Allotruxalis Rehn and Eutryxalis Bruner (Orthoptera: Acrididae: Hyalopterygini). Caldasia, 25: 381-402.

dos Santos, A.C. \& M.F. Vieira, 1999. O ciclo de vida de Cornops aquaticum (Orthoptera: Acrididae: Leptysminae) em condições naturais no laboratório. Anais da VIII Jornada de Iniciação Científica, INPA. Manaus, Brazil: 37-40.

Eades, D.C., D. Otte, M.M. Cigliano \& H. Braun, 2014. Orthoptera Species File. Version 5.0/5.0. [12/mar/2014]. <http:// Orthoptera.SpeciesFile.org $>$.

Elgueta, M., A. Camousseight \& C.S. Carbonell, 1999. Catálogo de Orthoptera (Insecta) de Chile. Publicación ocasional - Museo Nacional de Historia Natural (Chile), 54: 1-60.

Escalante G., 1979. Nota sobre saltamontes acridoideos del AltoUrubamba, Cusco. Revista Peruana de Entomología, 22: 113114.

Ferreira, S.A. \& J. Vasconcellos-Neto, 2001. Ecology, behavior and bionomics of Cornops aquaticum in Poconé, Brazil. Neotropical Entomolology, 30: 523-533.

Flook, P.K. \& C.H.F. Rowell, 1997. The phylogeny of the Caelifera (Insecta, Orthoptera) as deduced from mtRNA gene sequences. Molecular Phylogenetics and Evolution, 8: 89103.

Fontana P., F.M. Buzzetti \& R.P. Marino Perez, 2008. Chapulines, langostas, grillos y esperanzas de México. Guía fotográficagrasshoppers, locusts, crickets and katydids of Mexico. Photogra- phic Guide. wba Handbooks, 1. Verona, 272p.

Franceschini, M.C., J. Adis, A.P. Neiff \& M.L. Wysiecki, 2007. Fenologia de Cornops aquaticum (Orthoptera: Acrididae) en un camalotal de Eichhornia azurea (Pontederiaceae) en Argentina. Amazoniana, XIX: 149-158.

Franceschini, M. C., S. Capello, M.G. Lhano, J. Adis \& M.L. Wysiecki, 2005. Morfometria de los estádios ninfales de Cornops aquaticum Bruner (1906) (Acrididae: Leptysminae) en Argentina. Amazoniana, XVIII: 373-386.

Franceschini, M.C., J.J. Neiff \& C. Amédégnato, 2011. The Oxycaryum cubense floating mat as refuge of Cornops aquaticum (Orthoptera: Acrididae), a new control agent of the water hyacinth. Studies on Neotropical Fauna and Environment, 46: 203-210.

Gallo, D., O. Nakano, S. Silveira Neto, R.P.L. Carvalho, G.C. Baptista, J.R.P. Parra, R.A. Zucchi, S.B. Alves, J.D. Vendramim, L.C. Marchini, J.R.S. Lopes, \& C. Omoto. 2002. Entomologia Agrícola. SEALQ, Piracicaba, Brasil, 920 pp.

Gangwere, S.K. \& R.A. Ronderos, 1975. A synopsis of food selection in Argentine Acridoidea. Acrida, 4: 173-194.

Gerstaecker, A., 1873. Acridoidea nonnulla nova insigniora. Entomologische Zeitung, 34: 185-197.

Gerstaecker, A., 1889. Carakteristik einer Reihe bemerkensewerther orthopteren. Mittheilugen aus dem naturwissenschafteichen verein fur neu-vorpommern und Rügen in Greifswald, 20: 1-58.

Giglio-Tos, E., 1894. Viaggio dell dott. Alfredo Borelli nella Repubblica Argentina e nel Paraguay. VII Ortotteri. Bolletino dei Musei di Zoologia ed Anatonia Comparata della $\mathrm{R}$. Universita di Torino, 9: 1-46.

Giglio-Tos, E., 1895. Ortotteri del Paraguay, racolti dal Dr. J. Bohls. Zoologischen Jahrbüchern, 8: 804-818.

Giglio-Tos, E., 1897. Ortotteri raccolti nel. Darien dal Dr. E. Festa. III, Acrididae, Gryllidae. Bolletino dei Musei di Zoologia ed Anatomia Comparata de la R. Universita di Torino, 12: 1-10.

Giglio-Tos, E., 1898. Viaggio del Dr. Enrico Festa nella Republica dell'Ecuador e regioni vicine. IV, Ortotteri. Bolletino dei Mesei di Zoologia ed Anatomia Compaarata della R. Universitá di Torino, 13: 1-108.
Giglio-Tos, E., 1900. Viaggio del Dr. A.Borelli nel Matto Grosso e nel Paraguay. IV, Ortotteri. Bullettino dei Musei di Zoologia ed Anatomia Comparata della R.Universitá di Torino, 15: 1-8.

Greathead, D.J., 1963. A review of the insect enemies of Acridoidea (Orthoptera). Transactions of the Royal Entomological Society of London. 114: 437-51.

Guerra, W.D., P.C. Oliveira \& J.R. Pujol-Luz, 2012. Gafanhotos (Orthoptera, Acridoidea) em áreas de cerrados e lavouras na Chapada dos Parecis, Estado de Mato Grosso, Brasil. Revista Brasileira de Entomologia. 6: 228-239.

Guido, A.S. \& B.D. Perkins, 1975. Biology and Host Specifity of Cornops aquaticum (Bruner) (Orthoptera: Acrididae), a potencial biological control agent for waterhyacinth. Environmental Entomology, 4: 400-404.

Haan, C.W., 1835. Icones Orthoptorum. Abbildungen der hautflugen insecten Nürnberg. 1e. Lieferung (3 unnumbered pages and 4 plates).

Hayward, J.K., 1960. Insectos tucumanos prejuduciales. Revista Industrial y Agricola de Tucumán, 42: 3-144.

Hebard, M., 1923. Studies in the Dermaptera and Orthoptera of Colombia. Third Paper, Orthopterous family Acrididae. Transactions of the American Entomological Society. 49: 165-313.

Hebard, M., 1924. Studies in the Dermaptera and Orthoptera of Ecuador. Proceedings of the Academy of Natural Sciences of Philadelphia, 76: 109-248.

Hebard, M., 1925. Dermaptera and Orthoptera from the state of Sinaloa, Mexico. Part II Saltatorial Orthoptera. Transactions of the American Entomological Society. 51: 265-310.

Hebard, M., 1931. Die Ausbeute der deutschen Chaco-Expedition 1925-26. Orthoptera. Konowia, Zeitschrift für Systematische Insektenkunde. 10: 257-285.

Hebard, M., 1932. New species and records of Mexican Orthoptera. Transactions of American entomological Society, 58: 201-371.

Hebard, M., 1933a. Notes on Panamanian Dermaptera and Orthoptera. Transactions of the American Entomological Society. 59: 103-144.

Hebard, M., 1933b. Studies in the Dermaptera and Orthoptera of Colombia. Supplement to papers one to five. Transactions of the American Entomological Society. 59:13-67.

Hepper, H.C., 1945. Notes ecológicas, sistemáticas y zoogeográficas de acridios de la Argentina. Revista de la Sociedad Entomológica Argentina. 12: 280-298.

Hill, M.P. \& C.J. Cilliers, 1999. A review of the arthropod natural enemies, and factors that influence their efficacy, in the biological control of water hyacinth, Eichhornia crassipes (Mart.) Solms-Laubach (Pontederiaceae), in South Africa. African Entomology, 1: 103-112.

Hill, M.P. \& H. Oberholzer, 2000. How Safe Is the Grasshopper Cornops aquaticum for Release on Water Hyacinth in South Africa? Proceedings of the X International Symposium on Biological Control of Weeds. p. 349-356.

Hollier, J.A., 2010. An annotated catalogue of the primary type specimens of the Orthoptera (Insecta) species described by Johann Carl and Adolf Fritze. Revue Suisse de Zoologie. 117: 23- 44.

Hollier, J.A., 2012. An annotated list of the Orthoptera (Insecta) species described by Henri de Saussure, with an account of the primary type material housed in the Muséum d'histoire naturelle de Genève, part 3: The Acridoidea excluding the Acrididae: Oedipodinae. Revue Suisse de Zoologie. 119: 303339.

Hudson, W.H., 1895. The Naturalist in La Plata. London: Chapman and Hall.394p.

Jago, N.J., 1971. A review of the Gomphocerinae of the world, with a key to the genera (Orthoptera, Acrididae). Proceedings of the Academy of Natural Sciences of Philadelphia, 123: 205343 . 
Jago, N.J., 1989. The genera of Central and South American Grasshopper Family Proscopiidae (Orthoptera: Acridomorpha). EOS, 65: 249-307.

Kevan, D.K.McE., 1968. Pycnosarcini and additional tribe of romaleine Acrididae (Orthoptera). Revista de la Sociedade Uruguaya de Entomologia, 7: 66-71.

Kirby, W.E., 1890. On the empoyment of the names proposed for genera of Orthoptera previous to 1840. Scientific Proceedings of the Royal Dublin Society, 6: 556-597.

Kirby, W.E., 1910. A synonymic catalogue of orthoptera. Vol. 3. Orthoptera Saltatoria. Part. 2. (Locustidae, Acrididae). British Museum: London, 674p.

Kirby, W.E., 1914. Orthoptera, Acrididae. In: Shipley A.E. (ed.) The Fauna of British India. Taylor and Francis, London, 276 pp.

Klug, F., 1820. Proscopia, novum Insectorum Orthopterorum genus. Horae Physicae Berolinensis, p. 15-26.

Lange, C.E., 1992. Espectro hospedador natural y persistencia de Perezia dichroplusae Lange y Nosema locustae Canning (Protozoa: Microspora) en acridios argentinos (Orthoptera: Acrididae). Neotropica. 38: 65-74.

Lange, C.E., 1997. Viabilidad del acridicida Nosema locustae (Protozoa, Microspora) luego de almacenamiento prolongado. Revista de la Sociedad Entomológica Argentina. 56: 63-65.

Leitão, C.M., 1939. Estudio monográfico de los Procópidos. Revista del Museo de la Plata (N.S.), 1: 279-450.

Lhano, M.G., J. Adis, M.I. Marques \& L.D. Battirolla, 2005. Cornops aquaticum (Orthoptera, Acrididae, Leptysminae): aceitação de plantas alimentares por ninfas vivendo em Eichhornia azurea (Pontederiaceae) no Pantanal Norte, Brasil. Amazoniana, XVIII: 397-404.

Liana. A., 1980. Materiaux pour la connaissance des Proscopiidae (Orthoptera). Mitteilungen aus dem Hamburgischen Zoologischen Museum und Institut, 77: 229-260, illustr.

Liebermann, J., 1928[1927]. Preliminares para el estudio de los acridoideos argentinos. Anales de la Sociedad Científica Argentina, 104: 137-158.

Liebermann, J., 1929. Ocho especies de tucuras argentines con su definitiva posisión sistemática. Revista de la Sociedad Entomológica Argentina, 2: 179-180.

Liebermann, J., 1935. Catálogo sistematico y razonado de acridoideos argentinos. Gêneros: Prionolopha, Diedronotus, Elaeochlora y Chromacris (Orthoptera, Acrididae). Revista de la Sociedad Entomologica Argentina, 7: 25-50.

Liebermann, J., 1937. Los predatores vertebrados mas importantes da la Schistocerca paranaensis (Burm.) Lat. en la Republica Argentina y la necesidad de su protección. Revista Chilena de Historia Natural, 41: 49-57.

Liebermann, J., 1939a. Catálogo Sistemático y Biogeografico de Acridoideos Argentinos. Revista de la Sociedad Entomológica Argentina, 10: 125-230.

Liebermann, J., 1939b. Los Tropidacrini de la Región Neotropical. Phycis, 17: 589-600.

Liebermann, J., 1940. Una nueva especie de Acridoideo para la fauna argentina: Antiphon gallus Stål (Orth. Acrid. Cyrtacanthacr.Monach.). Revista dela Sociedad Entomologica Argentina, 10: 363-367.

Liebermann, J., 1941. Contribución a la zoogeografía, taxonomía y ecología de los acridoideos de Entre Rios. Publicacion del Ministerio de Hacienda, Justicia e Instruccion Publica de Parana Entre Rios, 41p.

Liebermann, J., 1943. Consideraciones sobre el género Cornopus Scudder (=Paracornopus Gigio-Tos) y su hallazgo en la Republica Argentina (Orth., Acrid., Cyrtacanth.). Revista de la Sociedad Entomológica Argentina, 12: 22-25, 1 pl.

Liebermann, J., 1945. Sobre una colección de acrididos (Orth. Acrididae) del Instituto Miguel Lillo. Acta Zoológica Lilloana, 3: 235-238.

Liebermann, J., 1947. Nuevos materiales acridicos de la colección del Instituto Oswaldo Cruz, con algunas observaciones sobre Episcopotettix Rehn, 1902. Revista Brasileira de Biologia, 7:
391-394.

Liebermann, J., 1948. Los Acridios de Santa Fe. Revista de la Sociedad Entomológica Argentina, 14: 55-114.

Liebermann, J., 1949. Sobre una colección de acridios argentinos del Naturhistorischen Museum de Viena (Orth. Acrid.). Publicaciones del Instituto de Sanidad vegetal, Ministerio de Agricultura Nacional, Republica Argentina, A 5: 1-12.

Liebermann, J., 1949. Exploración acridiológica en Corrientes. IDIA, 15: 14-15.

Liebermann, J., 1950. Sintesis de Sinipta, Stal, 1873, con la descripcion de una nueva especie argentina $S$. hectorisperonii (Orth. Acrid., Acridinae). Publicaciones del Instituto de Sanidad vegetal, Ministerio de Agricultura Nacional,, 6: 3-16.

Liebermann, J., 1951. Los acridoideos de Corrientes. IDIA, 4: 3948.

Liebermann, J., 1954. Los acridoideos de Catamarca y La Rioja. Publicaciones del Instituto de Sanidad Vegetal, Buenos Aires.

Liebermann, J., 1955. Primeira relação sistemática dos Acridoideos do Brasil. Memórias do Instituto Oswaldo Cruz, 53: 329-344.

Liebermann, J., 1958. Los Acridios de Formosa (Orth. Caelifera, Acridoidea).. Publicaciones del Instituto Nacional de Tecnologta Agropecuaria, 5: 3-24

Liebermann, J., 1965. Nuevos materiales homotípicos en la colección de acridios del Instituto de Patología Vegetal, Instituto Nacional de Technología Agropecuaria, INTA (Orthoptera). Physis, 25: 213-222

Liebermann, J., 1967. Seis nuevas notas colaterales sobre acridiologia Argentina (VI a XI). Physis, 26: 503-508.

Liebermann, J., 1968. Lista alfabética preliminar de las tucuras de la tribu Dichroplini de la región neotropical (Orthoptera: Acrididae). Revista de la Sociedad Entomológica Argentina, 30: $27-36$

Liebermann, J., 1969. El género Elaeochlora Stål, 1873, con la descripción de los alotipos de Elaeochlora trilineata (Serville) y Elaeochlora viridicata (Serville) (Orthotpera, Acrididae). Revista de la Sociedad Entomologica Argentina, 31: 81-91.

Liebermann, J., 1971. El alotipo de Dichroplus amoenus (Stål) y algunas consideraciones sobre la especie (Orth. Acrididae). Anales de la Sociedad Científica Argentina, 191: 145-155.

Liebermann, J., 1972a. The current state of the locust an grasshopper problem in Argentina. Proceedings of the International Study Conference on Current and Future Problems of Acridiology, 191-198

Liebermann, J., 1972b. Identificación de tucuras recolectadas en 1971 por el Ministerio de Agricultura de Santa Fe en el Departamento de San Jerónimo. IDIA, 290: 6-8

Liebermann, J. \& R. Schiuma, 1946. Las "tucuras" más perjudiciales de nuestra agricultura y ganadería. Publicaciones del Instituto de Sanidad Vegetal, Buenos Aires, B 2: 1-63.

Lima, A.M.C., 1942. Sobre alguns gafanhotos do grupo Copiocerae (Acrididae, Cyrtacanthacrinae). Revista Brasileira de Biologia, 2: $57-65$.

Lockwood, J.A. \& A.V. Latchininsky, 2008. Philosophical justifications for the extirpation of non-indigenous species: the case of the grasshopper Schistocerca nitens (Orthoptera) on the Island of Nihoa, Hawaii. Journal of Insect Conservation, 12: 429-445.

Luna, G.C., J. E. Henry \& R. Ronderos, 1981. Infecciones experimentales y naturales con protozoos patógenos en acridios de la República Argentina (Insecta, Orthoptera). Revista de la Sociedad Entomológica Argentina, 40: 243247.

McNeill, J., 1897. Revision of the Truxalinae of North America. Proceedings of the Davenport Academy of Natural Sciences, 6: $179-274$

Marques, M.I., J. Adis, G.B. dos Santos \& M.G. Lhano, 2002. Ecology and taxonomy of terrestrial arthropods in the Brazilian Pantanal. The International Canopy Network 
Newsletter, 8: 6-7.

Marschall, A.F., 1836. Decas Orthopterorum Novorum. Zoologische Abhandlungen aus dem Annalen des Wiener Museums der Naturgeschichte, 1-2: 207-218.

Marshall, J.A., 1983. The orthopteroid insects described by Linnaeus, with notes on the Linnaean collection. Zoological Journal of the Linnean Society, 78: 375-396.

Mariottini, Y., M.L. de Wysiecki \& C.E. Lange, 2006. Desarrollo postembrionario de Ronderosia bergi (Orthoptera: Acrididae: Melanoplinae) bajo condiciones controladas. Revista de la Sociedad Entomológica Argentina, 65: 81-85.

Mariottini, Y.; M.L. de Wysiecki \& C.E. Lange, 2010. The biology and some population parameters of the grasshopper, Ronderosia bergi, under laboratory conditions. Journal of Insect Science, 10:92 (12p).

Mariottini, Y., M.L. de Wysiecki \& C.E. Lange, 2011. Seasonal occurrence of life stages of grasshoppers (Orthoptera: Acridoidea) in the southern Pampas, Argentina. Zoological Studies, 50: 737-744.

Matiotti da Costa, M.K. \& G. Silva Carvalho, 2006. Revisão dos gêneros Sitalces, Eusitalces e Parasitalces (Orthoptera, Acrididae, Abracrini) e descrição de tres novos gêneros. Revista Brasileira de Entomologia, 50: 137-152.

Matiotti da Costa, M.K., A. Ferrari \& G. Silva Carvalho, 2007. Análise cladística e biogeográfica dos gêneros do grupo Sitalces (Orthoptera, Acrididae, Abracrini). Iheringia Série Zoologia, 97: 383-391.

Mesa, A., A. Ferreira \& C.S. Carbonell, 1982[1983]. Cariología de los acridoideos neotropicales: estado actual de su conocimiento y nuevas contribuciones. Annales de la Société Entomologique de France, 18: 507-526.

Mesa, A., P.C. García-Novo, C.B. Portugal \& A.R. Miyoshi, 2004. Karyology of species belonging to the genera Agriacris Walker 1870 and Staleochlora (Roberts \& Carbonell 1992) with some considerations of romaleid phallic structures (Orthoptera, Acridoidea). Journal of Orthoptera Research, 13: 15-18.

Mesa, A. \& L.C. Zolessi, 1968. Descripción y observaciones bioecológicas sobre una nueva especie de Scotussa (Orthoptera: Acrididae). Revista de La Sociedad Uruguaya de Entomologia, 77: 4-19.

Michel, A.A. \& E.S. Teisaire, 1996. Cronología del desarrollo embrionario normal de Baeacris punctulatus (Thunberg) (Orthoptera: Acrididae). Revista Chilena de Entomologia, 23: 29-41.

Mitchell, D.S. \& P.A. Thomas, 1972. Ecology of water weeds in the neotropics; an ecological survey of the aquatic weeds Eichhornia crasspipes and Salviniaspecies, and their natural enemies in the neotropics. UNESCO Technical Papers in Hydrology Series, 12: 5op.

Oberholzer, I.G. \& M.P. Hill, 2001. How safe is the grasshopper Cornops aquaticum for release on water hyacinth in South Africa, p. 82-88. In: Julien, M. H., M.P. Hill, T. D. Center \& D. Jianqing (eds.) Biological and integrated control of water hyacinth, Eichhornia crassipes. ACIAR Proceedings, 102p.

Olivier, A.G., 1791. Criquet, p. 104-236. In: Encyclopedie Methodique, Histoire Naturelle, Insectes, Paris, Panckoucke, Imprimeur, Libraire, Tome 6, 704p.

Otte, D., 1979. Revision of the Grasshopper Tribe Orphulellini (Gomphocerinae, Acrididae). Proceedings of the Academy of Natural Sciences of Philadelphia, 131: 52-88.

Otte, D., 1981. Acrididae: Gomphocerinae and Acridinae. North American Grasshoppers. 1: 274p.

Otte, D, 1995. Grasshoppers [Acridomorpha] D. Orthoptera Species File, 5: $630 \mathrm{pp}$.

Otte, D. \& N.J. Jago, 1979. Revision of the grasshopper genera Silvitettix and Compsacris (Gomphocerinae, Acrididae). Proceedings of the Academy of Natural Sciences of Philadelphia, 131: 257-288.

Passerin d'Entrèves, P., 1981. Cataloghi. IV - Collezioni Ortotterologiche del Museo di Zoologia dell'Università di Torino, $127 \mathrm{pp}$.
Paradeda, M.G., A.M. Bentos-Pereira \& A. Novello, 2008. Comparative study of the grasshopper Zoniopoda tarsata (Romaleide) and Paulinia acuminata (Pauliniidae) based on chromosome and C-banding analysis. Caryologia, 61(2): 123127.

Perty, M., 1834. Delectus animalium articulatorum quae in itinere per Brasiliam, annis MDCCCXVII - MDCCCXX, jussu et auspiciis Maximiliani Josephi I., Bavariae Regis augustissimi peracto collegerunt Dr. J.B. de Spix et Dr. C.F.Ph. de Martius. Monachii 1830-34, vol. 24, iii + 224p.

Pictet, A. \& H. Saussure, 1887. Catalogue d'Acridiens. I. Bulletin de la Société Entomologique Suisse, 7: 331-376.

Piza Jr., S.T., 1979. Proscopiidae novae brasiliensis (Orthoptera). Revista Brasileira de Entomologia, 23: 85-87.

Prinzessin von Bayern, T., 1900. Die von ihrer Königl. Hoheit Prinzessin Therese von Bayern auf einer Reise in Südamerika gesammelten Insekten. II. Orthopteren. Berliner Entomologische Zeitschrift, 45: 253-268.

Rehn, J.A.G., 1902. A contribution to the knowledge of the Orthoptera of Mexico and Central America. Transactions of the American Entomological Society, 29: 1-34.

Rehn, J.A.G., 1903. Notes on the West Indian Orthoptera, with a list of the species known from the island of Porto Rico. Transactions of the American Entomological Society, 29: 129-136.

Rehn, J.A.G., 1904. Notes on Orthoptera from Northern and Central Mexico. Proceedings of the Academy of Natural Sciences of Philadelphia, 56: 513-549.

Rehn, J.A.G., 1905a. Notes on the Orthoptera of Costa Rica, with descriptions of new species. Proceedings of the Academy of Natural Sciences, Philadelphia, 57: 790-843.

Rehn, J.A.G., 1905b. A contribution to the knowledge of the Acrididae (Orthoptera) of Costa Rica. Proceedings of the Academy of Natural Sciences, Philadelphia, 57: 400-454.

Rehn, J.A.G., 1905c. Notes on a small collection of Orthoptera from the Lesser Antilles, with the description of a new species of Orphulella. Entomological News, 16: 173-182.

Rehn, J.A.G., 1906. Studies in South and Central American Acridinae (Orthoptera), eight descriptions of a new genus and six new species. Proceedings of the Academy of Natural Sciences of Philadelphia, 58: 10-50.

Rehn, J.A.G., 1907. Non-saltatorial and acridoid Orthoptera from Sapucay, Paraguuai. Proceedings of the Academy of Natural Sciences of Philadelphia, 59: 151-192.

Rehn, J.A.G., 1909. On Brazilian Grasshoppers of the subfamilies Pyrgomorphinae and Locustinae (Acrididae of Authors). Proceedings of the United States National Museum, 36: 109163.

Rehn, J.A.G., 1913. Descriptions and records of South American Orthoptera, with the description of a new subspecies from Clarion Island. Proceedings of the Academy of Natural Sciences of Philadelphia, 63: 82-113.

Rehn, J.A.G., 1915. A further contribution to the knowledge of the Orthoptera of Argentina. Proceedings of the Academy of Natural Sciences, Philadelphia, 67: 270-292.

Rehn, J.A.G., 1916. The Stanford expedition to Brazil. 1911, Dermaptera and Orthoptera I. Transactions of the American Entomological Society, 42: 215-308.

Rehn, J.A.G., 1918. On Dermaptera and Orthoptera from Southeastern Brazil. Transactions of the American Entomological Society, 44: 181-222.

Rehn, J.A.G., 1920. Records and descriptions of Brazilian Orthoptera. Proceedings of theAcademy of Natural Sciences of Philadelphia, 72: 214-293.

Rehn, J.A.G., 1944. A revision of the locusts of the group Hyalopteryges (Orthoptera, Acrididae, Acridinae). Transactions of the American Entomological Society, 70: 181234.

Rehn, J.A.G., 1955. The species of the genus Procolpia (Acrididae, Romaleinae). Transactions of the American Entomological Society, 81: 37-57. 
Rehn, J.A.G. \& D. Eades, 1961. The tribe Leptysmini (Orthoptera: Acrididae: Cyrtacanthacridinae) as found in North America and Mexico. Proceedings of the Academy of Natural Sciences of Philadelphia, 113: 81-134.

Ribeiro, R.C., W. de Paulo Lemos, J.C.M. Poderoso, T.G. Pikart \& J.C. Zanuncio, 2013. New record of grasshopper (Orthoptera: Acrididae \& Romaleidae) defoliators and population dynamics of insects on crops of Heliconia spp. in the Amazon. Florida Entomologist, 96: 225-228.

Richman, D.B., D.C. Lightfoot, C.A. Sutherland \& J.W.H. Ferguson. 1993. A manual of the grasshoppers of New Mexico, Orthoptera: Acrididae and Romaleidae. New Mexico State University Cooperative Extension Service Handbook, 7: 1-112, illustr.

Riede, K., 1987. A comparative study of mating behaviour in some neotropical grasshoppers (Acridoidea). Ethology, 76: 265-296.

Rivera García, E., 2006. An annotated checklist of some orthopteroid insects of Mapimi Biosphere Reserve (Chihuahuan desert), Mexico. Acta Zoológica Mexicana (nueva serie), 22: 131-149.

Roberts, H.R., 1937. Studies on the family Acrididae (Orthoptera) of Venezuela. Proceedings of the Academy of Natural Sciences, Philadelphia, 89: 343-368.

Roberts, H.R., 1975. A revision of the genus Cylindrotettix including new species (Orthoptera, Acrididae, Leptysminae). Proceedings of the Academy of Natural Sciences of Philadelphia 127: 29-43.

Roberts, H.R., 1977[1978]. A revision of the tribe Leptysmini except the genus Cylindrotettix (Orthoptera: Acrididae: Leptysminae). Proceedings of the Academy of Natural Sciences, Philadelphia, 129: 33-69.

Roberts, H.R. \& C.S. Carbonell, 1979. A revision of the genera Stenopola and Cornops (Orthoptera, Acrididae, Leptysminae). Proceedings of the Academy of Natural Sciences of Philadelphia, 131: 104-130.

Roberts, H.R. \& C.S. Carbonell, 1981. Revision of the Neotropical genus Abracris and related genera (Orthoptera, Acrididae, Ommatolampinae). Proceedings of the Academy of Natural Sciences of Philadelphia, 133: 1-14.

Roberts, H.R. \& C.S. Carbonell, 1982. A revision of the grasshopper genera Chromacris and Xestotrachelus (Orthoptera, Romaleidae, Romalienae). Proceedings of the California Academy of Sciences, 43: 43-58.

Roberts, H.R. \& C.S. Carbonell, 1992. Revision of the genera Agriacris Walker 1870 and Staleochlora Nov. (Orthptera, Romaleidae). Journal of Orthoptera Reearch, 1: 75-106.

Ronderos, R.A., 1964. Contribución al conocimiento del complejo fálico en especies de los géneros Aleuas Stål y Dichroplus Stål de la Provincia de Buenos Aires (Orthoptera, Acrididae). Revista de Investigaciones Agropecuarias, Ser. 5, Patología Vegetal, 1: 55-96.

Ronderos, R.A., 1977[1978]. Notas para una revisión de la subfamilia Ommexechinae. 8. El género Ommexecha Serville (Orthoptera: Acridiomorpha). Revista de la Sociedad Entomológica Argentina, 36: 97-111.

Ronderos, R.A., 1985. Consideraciones sobre la biogeografía de los Melanoplinae en Sudamérica (Orthoptera: Acrididae). Proceedings, Triennial Meeting Pan-American Acridological Society, 3: 53-54.

Ronderos, R.A., C.S. Carbonell \& A. Mesa, 1968. Revisión de las especies del gênero Dichroplus Stål, Del grupo Elongatus (Orthoptera, Acrididae, Catantopinae). Revista Del Museo de La Plata, (N.S. Zoologia), 10: 271-325.

Ronderos, R.A. \& M.M. Cigliano, 1991. The Andean Dichroplini: cladistic analysis with description of Keyacris n.gen. and Ponderacris n.gen. (Orthoptera, Acrididae, Melanoplinae). Transactionsof the American Entomological Society, 117: 167-191.

Ronderos, R.A. \& C.S. Carbonell, 1994. Sobre el género mexicano Pedies Saussure, y las especies sudamericanas atribuidas al mismo (Orthoptera: Acrididae, Melanoplinae). Revista de la Sociedad Entomológica Argentina, 53: 83-99.

Rowell, C.H.F., 1978. Food plant specificity in neotropical rainforest acridids. Entomologia Experimentalis et Applicata, 24: 651-662.

Rowell, C.H. F., 1983. Osmilia flavolineata. Pages 750-751 in D. H. Janzen, editor. Costa Rican natural history. Univer- sity of Chicago Press, Chicago, Illinois.

Rowell, C.H.F., 2013. The Grasshoppers (Caelifera) of Costa Rica and Panama, $611 \mathrm{pp}$.

Rowell, C.H.F. \& C.S. Carbonell, 1977. Baeacris talamancensis gen. and sp. nov. (Acrididae, Melanoplinae) a neotropical montane grasshopper; its implications for the origin of the Dichroplini and the Costa Rican paramo. Acrida, 6: 55-74.

Rowell, C.H.F. \& R.A. Behrstock, 2012. Additions to the acridoid grasshopper fauna of El Salvador. Journal of Orthoptera Research, 21: 235-243.

Sánchez, N.E. \& M.L. de Wysiecki. 1993. Abundancia y diversidad de acridios en pasturas dela Provincia de La Pampa, Argentina. Revista de Investigaciones Agropecuarias, 24: 29-39.

Santoro, H.F., 1976. Calculo de la cantidad de estadios ninfales o de la edad, por el análisis de la antena del adulto o de los estadios inmaduros previos, en diversas especies de acridios. Revista de Investigaciones Agropecuarias, 12: 1-6.

Santoro, H.F. \& A.E. Carames, 1973. Antenitos que determinan las variaciones cuantitativas postembrionales en la antena de doce especies de acridios de la Argentina (Insecta, Orthoptera). Revista de Investigaciones Agropecuarias, 10 197-209.

Saussure, H.L.F., 1859. Orthoptera nova americana (Diagnoses præliminares). Revue et Magasin de Zoologie pure et Appliquée (Guérin Meneville), 2éme Serie 11:59-63, 201212, 315-317, 390-394. (note: Acrididae in p. 390-394, Sept. 1859.)

Scudder, S.H., 1869. A study of the gigantic lobe-crested grasshoppers of South and Central America. Proceedings of the Boston Sciety of Natural History, 12: 345-355.

Scudder, S.H., 1875. Notes on Orthoptera from northern Peru collected by Professor James Orton. Proceedings of the Boston Society of Natural History, 17: 257-282.

Scudder, S.H., 1890. Some genera of the Oedipodidae rescued from the Tryxalidae. Psyche, 5: 431-442.

Scudder, S.H., 1899. The Orthopteran genus Schistocerca. Proceedings of the American Academy of Arts and Sciences, 34: $441-476$

Schiuma, R., 1951. Los estudios biológicos como base fundamental para la lucha contra la tucura. Ciencia e Investigación, 7: 1015 .

Serville, J.G.A., 1831. Revue methodique des insectes de l'ordre des Orthoptéres. Annales des Sciences Naturelles, 22: 28-65, 134-167, 262-292.

Serville, J.G.A., 1839. Histoire naturelle des insectes Orthoptéres. Collection des suites a Buffon, Paris, xvii + 776p., Atlas, 4 pp., $+14 \mathrm{pl}$.

Silveira-Guido, A., J.F. Carbonell-Bruhn, O. Nuñez \& E. Valdes, 1958. Investigaciones sobre Acridoideos del Uruguay: sistemática, morfología, citología, economía, habitat, ciclo biológico, costumbres, ecología, geografía, enemigos naturales y control. Montevideo, Cátedra de entomología. Facultad de Agronomía. Universidad de la República, 485 p.

Silveira-Guido, A., 1975. Biology and host specificity of Cornops aquaticum (Bruner) (Orthoptera: Acrididae), a potential biological control agent for waterhyacinth. Environmental Entomology, 4: 400-404.

Song, H., 2004. Revision of the Alutacea Group of genus Schistocerca (Orthoptera: Acrididae: Cytracanthacridinae). Annals of the Entomological Society of America, 97: 420436.

Sperber, C.F., 1996. Field diet of the grasshopper Abracris dilecta Walker (Orthoptera, Acrididae). Revista Brasileira de Zoologia, 13: 127-135

Sperber,C.F.\&F.S.Lopes,1995.Padrõesdeherbivoriadogafanhoto 
Cornops aquaticum (Bruner, 1906) (Orthoptera: Acrididae) em Eichhornia azurea (Sw.) Kunth. (Pontederiaceae). Libro de Resúmenes del III Congreso Latinoamericano de Ecología. Venezuela, 21-20.

Stål, C., 1860. Orthoptera species nova descrpsit. p. 299-350. In: Kongliga Svenska Fregatten Eugenies Resa omkring jorden ...etc. vol 2, Zoologi, part 1, Insecta.

Stål, C., 1873. Recencio orthopterorum. Revue critique des Orthopteres déscrits par Linné, De Geer et Thunberg. Norstedt \& Soner, Stockholm, 105p.

Stål, C., 1878. Systema acridiodeorum. Essai d'une systematisation des acridoidées. Bihang till Kongliga Svenska VetenskapsAkademiens Handlingar, 5: 1-100.

Stoll, C., 1813. Naturlijke en naar leven nauwerkeurige gekleurde afbeeldingen en beschrijvigen der spoken, wandelende bladen, zabelspringhanen, krekels, trekspringhaanen en kakkerlaken, in alle vier deelen del werwld Europa, Asia, Afrika en Amerika. Representation exactement colorée d'aprés nature des spectres ou phasmes, des mantes, des sauterelles, des grillons, des criquets et des blattes Qui se trouvent dans les quatre parties du monde, l'Europe, l'Asie, l'Afrique et l'Amerique. Amserdam, J.C. Sepp et fils, vol. 1, 79 pp., 25 pl.; vol. 2, $28+42$ pp., $13+23$ pl.

Thunberg, C.P., 1815. Acrydii descriptio. Nova Acta Regiae Societatis Scientiarum Uppsaliensis, 7: 157-162.

Thunberg, C.P., 1824. Grylli monographia, illustrata. Mémoiers de l'Académie Impériale des Sciences de St. Pétersbourg, 3: 390-430.

Thunberg, C.P., 1827. Truxalis, insecti genus illustratm. Nova Acta Regiae Societatis Scientiarum Uppsaliensis, 9: 76-88.

Turk, S.Z., 1980. Acridios del NOA4. Contribución al conocimiento de huevos, desoves y habitos de postura de algunas especies de tucuras (Orthoptera: Acridoidea), de la Provincia de Tucuman. 2. Acta Zoológica Lilloana, 36: 122-130.

Turk, S.Z., 1981. Acridoideos del NOA. 5. Ciclo del vida y aspectos ecológicos en Osmilia violacea (Th.) (Acrididae, Ommatolampinae). Acta Zoológica Lilloana, 36: 53-59.

Turk, S.Z., 1993. Sobre la peculiar modalidad de cópula en Cornops frenatum cannae Roberts \& Carbonell (Acrididae: Leptysminae). Acta Zoológica Lilloana, 43: 463.

Turk, S.Z. \& M. Barrera, 1976. Acridios Del NOA. 1. Estúdios biológicos, morfométricos y aspectos ecológicos de Chromacris speciosa (Thunberg) (Acrididae, Romaleinae).
Acta Zoológica Lilloana, 32: 121-145.

Turk, S.Z. \& A.L. Aquino, 1996. Acridoideos del NOA VIII: nuevo aporte a la bioecologia y distribución del género Cornops Stål: Cornops paraguayense (Br.) (Acrididae: Leptysminae: Tetrataeniini). Acta Zoológica Lilloana, 43: 427-432.

Uvarov, B.P., 1925[1924]. Notes on the Orthoptera in the British Museum 4. Identification of the types of Acrididae preserved in the Museum. Transactions of the Entomological Society of London, 3-4: 265-301.

Uvarov, B.P., 1966. Grasshoppers and locusts. A handbook of general Acridology. v. 1. Cambridge University Press, London, 481p.

Vickery, V.R. \& D.K.M. Kevan, 1983. A monograph of the orthopteroid insects of Canada and adjacent regions. Memoirs of the Lyman Entomological Museum and Research Laboratory, 13: I-IV, i-xxii, 1-679, pl.1, I-VIII.

Vieira, M.F. \& A.C. Santos, 2003. Duracção do ciclo de vida de Cornops aquaticum (Bruner, 1906) (Orthoptera: Acrididae: Leptisminae) e aspectos de seu comportamento alimentar na Amazonia Central. Acta Amazonica, 33: 711-714.

Villalobos, L.C. de, 1980[1981]. Habitos alimentarios y morfologia mandibular de 19 especies de Melanoplinae (Orthoptera, Acrididae). Revista de la Sociedad Entomológica Argentina, 39: 325-333.

Virla de Arguello, N.E., 1978. Estudio de las mandíbulas y análisis de las heces en 15 especies de tucuras colectadas en alfalfares de Córdoba. Revista de la Sociedad Entomológica Argentina, 36: 113-123.

Walker, F., 1870. Catalogue of the specimens of Dermaptera Saltatoria in the collection of the British Museum. British Museum (Natural History) 3: 425-604, 4:605-809, 5:811850.

Yin, X.C., J. Shi \& Z. Yin, 1996. Synonymic Catalogue of Grasshoppers and their Allies of the World (Orthoptera: Caelifera), 1266p.

Zolessi, L.C., 1956. Observaciones sobre Cornops aquaticum Br. (Acridoidea, Cyrtacanthacr.) en el Uruguay, Revista de la Sociedad Uruguaya de Entomologia, 1: 3-28.

Recebido em: 24/05/2013

Aceito em: 24/05/2013

\section{Como citar este artigo:}

Assis-Pujol, C.V. \& J.R. Pujol-Luz, 2014. Checklist de Caelifera (Acridoidea e Eumastacoidea) do Estado do Rio de Janeiro (Orthoptera). EntomoBrasilis, 7 (2): 134-150.

Acessível em: doi:10.12741/ebrasilis.v7i2.354
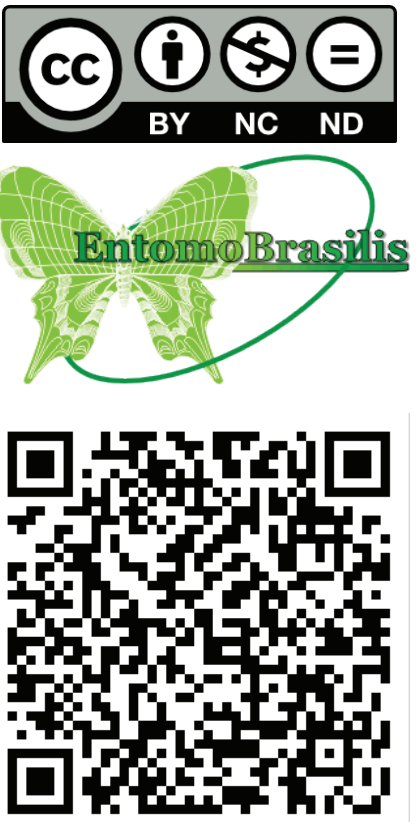